\title{
Spacecraft formation dynamics and design
}

\author{
V.M. Guibout* and D.J. Scheeres ${ }^{\dagger}$ \\ The University of Michigan, Ann Arbor, MI 48109
}

\begin{abstract}
This paper applies previous research on the solutions of two-point boundary value problems to spacecraft formation dynamics and design. The underlying idea is to model the motion of a spacecraft formation as a Hamiltonian dynamical system in the vicinity of a reference solution. Then we can analytically describe the nonlinear phase flow using generating functions found by solving the Hamilton-Jacobi equation. Such an approach is very powerful and allows one to study any Hamiltonian dynamical systems independent of the complexity of its vector field, and to solve any two-point boundary value problem using only simple function evaluations. We present the details of our approach through the study of a non-trivial example, the design of a formation in Earth orbit. For our analysis the effect of the $J_{2}$ and $J_{3}$ gravity coefficients are taken into account and the reference trajectory is chosen to be an orbit with high inclination $(i=\pi / 3)$ and eccentricity $(e=0.3)$. Two missions are considered. First, given several tasks over a one month period modeled as configurations at given times, we find the optimal sequence of reconfigurations to achieve these tasks with minimum fuel expenditure. Next we use our theory to find stable configurations such that the spacecraft stay close to each other for an arbitrary but finite period of time. Both of these tasks are extremely difficult using conventional approaches, yet are simple to solve in our approach.
\end{abstract}

\section{Introduction}

Several missions and mission statements have identified formation flying as a means for reducing cost and adding flexibility to space-based programs. However, such missions raise a number of technical challenges as they require accurate dynamic models of the relative motion and control techniques to achieve formation reconfiguration and formation maintenance. There is a large literature on spacecraft formation flight that we will not attempt to survey in a systematic manner. On one hand we find articles that focus on analytical studies of the relative motion, and on the other hand there are a large class of articles that develop numerical algorithms that solve specific reconfiguration and formation keeping problems. Theoretical studies require a dynamical model for the relative motion that is accurate and tractable. For that reason the Clohessy-Wilshire (CW) equations, Hill's equations or Gauss variational equations have often been used as a starting point. Using the CW equations, Hope and Trask ${ }^{1}$ study hover type formation flying about the Earth, Vadali, Vaddi and Alfriend ${ }^{2}$ look at periodic relative motion about the Earth, Gurfin and Kasdin, ${ }^{3}$ and Scheeres, Hsiao and Vinh $^{4}$ focus on formation keeping, Howell and Marchand, ${ }^{5}$ and Vadali, Bae and Alfriend ${ }^{6}$ analyze relative motion in the vicinity of the libration points and Vaddi, Alfriend and Vadali ${ }^{7}$ study the reconfiguration problem using impulsive thrusts. However, for a large class of orbits these approximations do not hold $J_{2}$ effects as well as non-circular reference trajectory should be taken into account for low Earth orbits and an elliptic orbit for the primary should be considered to study the dynamics at the Libration points. As a result, past researchers have modified the $\mathrm{CW}$ equations in order to take the $J_{2}$ gravity coefficient into consideration. These improved equations have been widely used; Alfriend and Schaub ${ }^{8}$ study periodic relative motion and Lovell, Horneman, Tollefson and Tragesser ${ }^{9}$ analyze formation reconfiguration with impulsive thrusts. The non-impulsive thrust problem is usually solved using optimal control theory (although there are some exceptions, for instance F.Y. Hsiao and D.J. Scheeres ${ }^{10}$ and I. Hussein, D.J. Scheeres and D. Hyland ${ }^{11}$ ), and if the dynamical model is tractable then analytical solutions for the feedback control law may be found $\left(\right.$ see Mishne ${ }^{12}$ ). These analytical approaches allow one to perform qualitative analysis and provide insight

*Graduate Student Research Assistant, PhD Candidate, Department of Aerospace Engineering, guibout@umich.edu

†Associate Professor, Department of Aerospace Engineering, Associate Fellow AIAA, scheeres@umich.edu 
into the dynamics of the relative motion. However they cannot be used for actual mission design (although there are some exceptions ${ }^{13}$ ). Indeed, they have inherent drawbacks: they neglect higher order terms in the dynamics and their domain of validity in phase space is very restricted and difficult to quantify. In addition, methods based on the state transition matrix tend to be valid only over short time spans. On the other hand, numerical algorithms have been developed to design spacecraft formations using the true dynamics. Koon, Marsden, Masdemont and Murray ${ }^{14}$ use Routh reduction to reduce the dimensionality of the system and then develop an algorithm based on the use of the Poincaré map to find pseudo-periodic relative motion in the gravitational field of the Earth (including the $J_{2}$ gravity coefficient only), Xu and Fitz-Coy ${ }^{15}$ and Avanzini, Biamonti and Minisci ${ }^{16}$ study formation maintenance as a solution to an optimal control problem that they solve using a genetic algorithm and a multi-objective optimization algorithm respectively. Even though these methods use the exact dynamics and therefore can be used to solve a specific reconfiguration or formation maintenance problem, they fail (except ${ }^{14}$ ) to give insight on the dynamics. In addition, as noticed by Wang and Hadaegh, ${ }^{17}$ formation reconfiguration design is a combinatorial problem. As a result the algorithms mentioned above are not appropriate for reconfiguration design as they require excessive computation (to reconfigure a formation of $N$ spacecraft, there are $N$ ! possibilities in general).

The method we expose in this paper directly tackles these issues and should be viewed as a semi-analytic approach, since it consists of a numerical algorithm whose output is a polynomial approximation of the dynamics. As a consequence, we are able to use a very accurate dynamical model and to obtain tractable expressions describing the relative motion. A fundamental difference with previous studies is that we describe the relative motion, i.e., the phase space in the vicinity of a reference trajectory, as two-point boundary value problems whereas it is usually described as an initial value problem. Such a description of the phase space is very natural and convenient, for instance the reconfiguration problem and the search for periodic formations can be naturally formulated as two-point boundary value problems.

In the present paper, to showcase the strength of our method, we have chosen to study two challenging mission designs.

1. We first consider a spacecraft formation about an oblate Earth (the $J_{2}$ and $J_{3}$ gravity coefficients are taken into account) that must achieve 5 missions over a one month period. For each mission the formation must be in a given configuration $C_{i}$ that has been specified beforehand, and we wish to minimize the overall fuel expenditure. The configurations $C_{i}$ are specified as relative positions of the spacecraft with respect to a specified reference trajectory (figure 1(a)). The $C_{i}$ 's may be fully defined or have one degree of freedom. In our example we require the spacecraft to be equally spaced on a circle centered on the reference trajectory at several epochs over the time period. The design of such a mission has several challenges:

- The dynamics are non-trivial and non-integrable,

- the reference trajectory has high eccentricity, high inclination and is not periodic,

- missions are planned a month in advance,

- in our specific example discussed here, 4 spacecraft must achieve 5 missions, if one assumes that the $C_{i}$ are fully defined there are 7,962,624 ways of satisfying the missions,

- the $C_{i}$ may be defined by holonomic constraints and have an additional degree of freedom.

2. Next we consider two problems, the initial deployment of a formation and the redesign of an already deployed formation. For both problems, given a reference trajectory we wish to place the spacecraft in its vicinity and ensure that they remain "close" to each other over an extended period of time (see figure $1(\mathrm{~b})$ ). This design is also very challenging because:

- the dynamics and the reference trajectory are non-trivial (as before),

- trajectories must not collide (except at the initial time for the deployment problem),

- high accuracy in the initial conditions is required for long-term integration.

In the following, we first introduce the dynamical model as well as the reference trajectory. We then briefly recall the theory developed in $^{18}$ for the solution of these problems. Finally, we study the missions discussed above. 


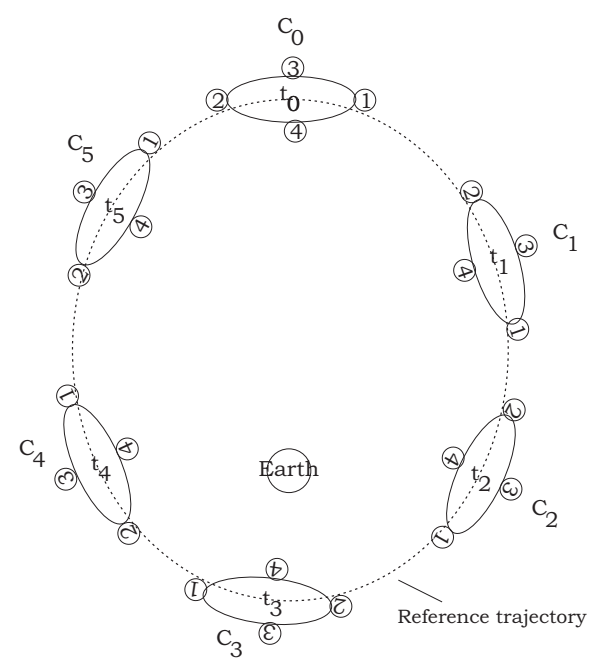

(a) At each $t_{i}$, spacecraft must be in the configuration $C_{i}$.

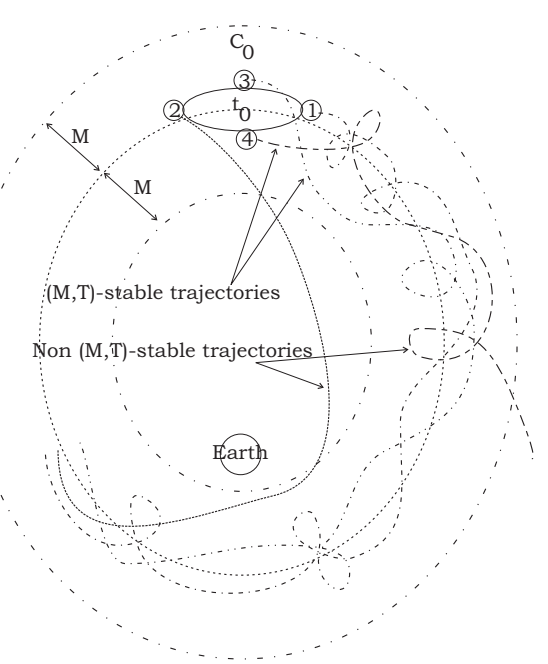

(b) Stable and non stable trajectories

Figure 1. Representations of the two designs we study: The multi-task mission and the search for stable configurations.

\section{Problem settings}

The motion of a satellite under the influence of the Earth modeled by an oblate sphere $\left(J_{2}\right.$ and $J_{3}$ gravity coefficients are taken into account) in the fixed coordinate system $(x, y, z)$ whose origin is the Earth center of mass is described by the following Hamiltonian:

$$
\begin{aligned}
H= & \frac{1}{2}\left(p_{x}^{2}+p_{y}^{2}+p_{z}^{2}\right)-\frac{1}{\sqrt{x^{2}+y^{2}+z^{2}}}[1- \\
& \left.\frac{R^{2}}{2 r_{0}^{2}\left(x^{2}+y^{2}+z^{2}\right)}\left(3 \frac{z^{2}}{x^{2}+y^{2}+z^{2}}-1\right) J_{2}-\frac{R^{3}}{2 r_{0}^{3}\left(x^{2}+y^{2}+z^{2}\right)^{2}}\left(5 \frac{z^{3}}{x^{2}+y^{2}+z^{2}}-3 z\right) J_{3}\right],
\end{aligned}
$$

where

$$
\begin{gathered}
G M=398600.4405 \mathrm{~km}^{3} \mathrm{~s}^{-2}, \quad R=6378.137 \mathrm{~km}, \\
J 2=1.082626675 \cdot 10^{-3}, \quad J 3=2.532436 \cdot 10^{-6},
\end{gathered}
$$

and all the variables are normalized ( $r_{0}$ is the radius of the trajectory at the initial time):

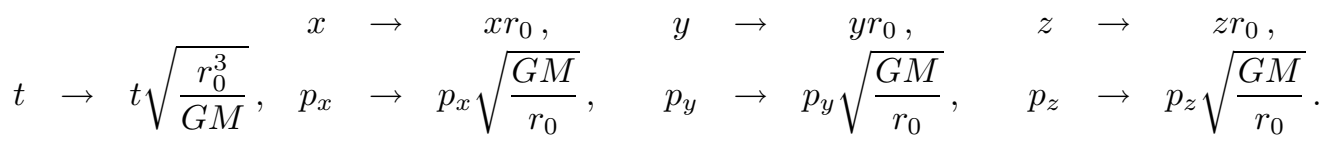

In the following, we consider a "reference" trajectory whose state is designated by $\left(q^{0}, p^{0}\right)$ and study the relative motion of spacecraft with respect to it. The reference trajectory is chosen to be highly eccentric and inclined, but any other choice could have been considered. At the initial time its state is:

$$
\begin{array}{ccc}
q_{x}^{0}=r_{p}, & q_{y}^{0}=0 \mathrm{~km}, & q_{z}^{0}=0 \mathrm{~km}, \\
p_{x}^{0}=0 \mathrm{kms}^{-1}, & p_{y}^{0}=\sqrt{\frac{G M}{\frac{1}{2}\left(r_{a}+r_{p}\right)}} \sqrt{\frac{r_{a}}{r_{p}}} \cos (\alpha) \mathrm{km} \mathrm{s}^{-1}, & p_{z}^{0}=\sqrt{\frac{G M}{\frac{G}{2}\left(r_{a}+r_{p}\right)}} \sqrt{\frac{r_{a}}{r_{p}}} \sin (\alpha) \mathrm{km} \mathrm{s}^{-1}, \\
\alpha=\frac{\pi}{3} \mathrm{rad}, & r_{p}=7,000 \mathrm{~km}, & r_{a}=13,000 \mathrm{~km} .
\end{array}
$$

Without the $J_{2}$ and $J_{3}$ gravity coefficients the reference trajectory would be an elliptic orbit with eccentricity $e=0.3$, inclination $i=\pi / 3 \mathrm{rad}$, argument of perigee $\omega=0$, longitude of the ascending node $\Omega=0$, semi-minor axis $r_{p}=7,000 \mathrm{~km}$, semi-major axis $r_{a}=13,000 \mathrm{~km}$ and of period $t_{p}=2 \pi \sqrt{\frac{1}{2^{3}} \frac{\left(r_{a}+r_{p}\right)^{3}}{r_{p}^{3}}}$ sec $\approx 2$ hours 45 min. The Earth oblateness perturbation causes (see Chobotov ${ }^{19}$ 
for more details) secular drifts in the eccentricity (due to $J_{3}$ ), in the argument of perigee (due to $J_{2}$ and $J_{3}$ ) and in the longitude of the ascending node (due to $J_{2}$ and $J_{3}$ ). In addition, all the orbit elements are subject to short and long period oscillations. In figure 2 and 3 , we plot the orbital elements for this trajectory as a function of time during a day (about 10 revolutions about the Earth) and over a month period. The symplectic implicit Runge-Kutta integrator built in Mathematica $^{\circledR}$ is used for integration of Hamilton's equations.

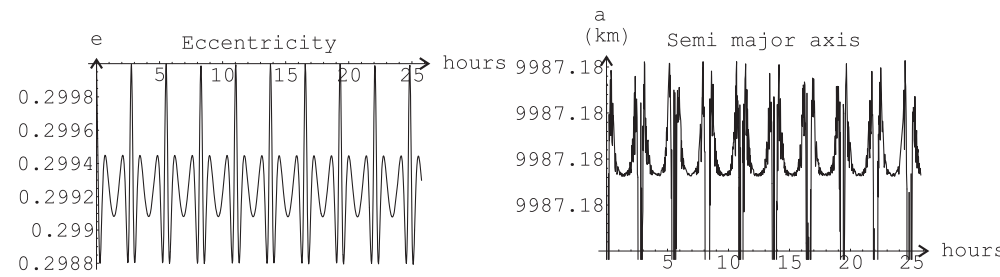

(a) (b)

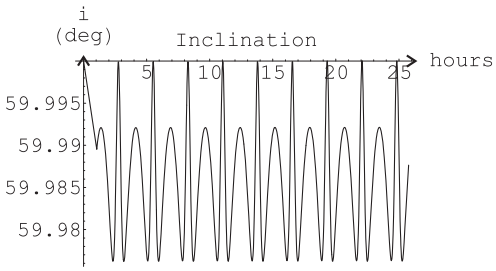

(c)

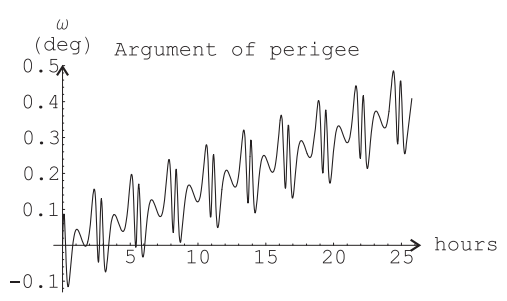

(d)

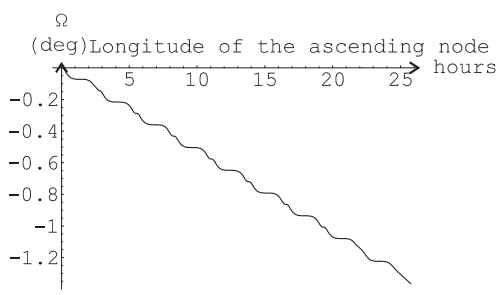

(e)

Figure 2. Time history of the orbital elements of the reference trajectory during a day

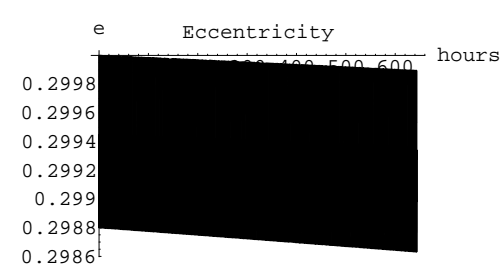

(a)

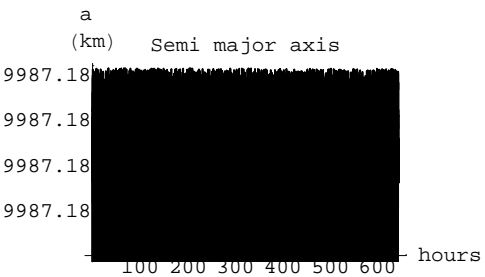

(b)

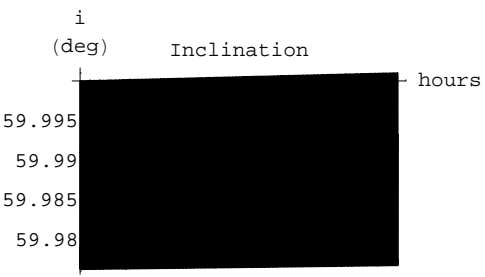

(c)

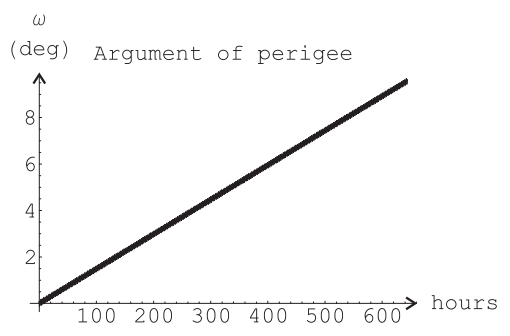

(d)

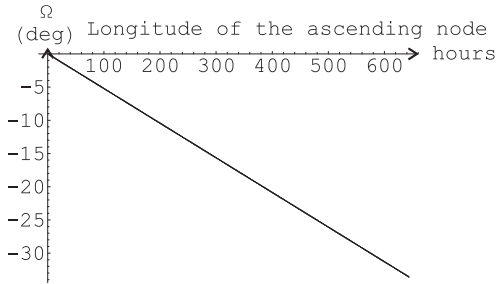

(e)

Figure 3. Time history of the orbital elements of the reference trajectory over a month period

\section{The algorithm}

To study relative motion about the above reference trajectory we use an algorithm whose output is an analytical description of the phase flow for the relative motion. It is based on previous studies by Guibout and Scheeres ${ }^{20,21}$ on the Hamilton-Jacobi theory and essentially consists of solving the Hamilton-Jacobi partial differential equation for an approximation of the generating functions for the phase flow transformation 
describing the relative motion.

\section{A. Relative motion}

The relative motion of a spacecraft whose state is $(q, p)$ moving in the Hamiltonian field defined by $H$ (Eq. $(2))$ with respect to the reference trajectory $\left(q^{0}(t), p^{0}(t)\right)$ is described by the Hamiltonian function ${ }^{20} H^{h}$ :

$$
H^{h}\left(X^{h}, t\right)=\sum_{p=2}^{\infty} \sum_{\substack{i_{1}, \cdots, i_{2 n}=0 \\ i_{1}+\cdots+i_{2 n}=p}}^{p} \frac{1}{i_{1} ! \cdots i_{2 n} !} \frac{\partial^{p} H}{\partial q_{1}^{i_{1}} \cdots \partial q_{n}^{i_{n}} \partial p_{1}^{i_{n+1}} \cdots \partial p_{n}^{i_{2 n}}}\left(q^{0}, p^{0}, t\right) X_{1}^{h^{i_{1}}} \ldots X_{2 n}^{h^{i_{2 n}}}
$$

where $X^{h}=(\Delta q, \Delta p), \Delta q=q-q^{0}, \Delta p=p-p^{0}$ and where we assume $X^{h}$ is small enough for the convergence of the series. Let us truncate the series in Eq. (5) in order to keep terms of order at most $N$. Then we say that the relative motion is described using an approximation of order $N$. Most past studies in the literature (CW, improved $\mathrm{CW}^{\mathrm{a}}$ and Hill's equations) consider an approximation of order 2, that is, a linear approximation of the dynamics. Although such an approximation is useful to obtain a first picture of the dynamical environment as well as qualitative results, it cannot be used for designing an actual formation for several reasons. First, nonlinear effects are usually not negligible, especially over a long time span. Second, linear effects may not be dominant over a short time span even though the Taylor series ${ }^{\mathrm{b}}$ converges $\left(\mathrm{see}^{22}\right.$ for a discussion on this issue). The algorithm we have developed tackles these issues, $N$ must be finite but can be as large as we want. There is no limit to the accuracy of the solution we obtain other than computer memory (as we will see in the next section, if a solution up to order $N-1$ is known, we need to solve $\frac{(N+5) !}{N ! 5 !}$ ordinary differential equations to obtain the $N$ th order).

\section{B. Solving boundary value problems}

The design of spacecraft formations can often be reduced to solving boundary value problems. Indeed, the reconfiguration problem is a position to position boundary value problem, ${ }^{20}$ the search for periodic configurations, that is spacecraft configurations that repeat themselves over time, may also be treated as a two-point boundary value problem ${ }^{23}$ and we will see in this paper how one may find stable formations, i.e., formations for which spacecraft naturally stay close to each other for a long time, as a solution to boundary value problems. Finally, if a maneuver is set up as an optimal control problem, the necessary conditions for optimality can in many cases be reduced to a Hamiltonian system with known boundary values, that is, a two-point boundary value problem. ${ }^{18,24}$

Traditionally used to analytically solve the equations of motion, ${ }^{25,26}$ the generating functions for the phase flow canonical transformation also allows one to solve two-point boundary value problems. ${ }^{21}$ Let us first consider a position $q_{0}$ to position $q$ boundary value problem and recall the generating function of the first kind $F_{1}\left(q, q_{0}, t\right)$ :

$$
\begin{aligned}
p_{i} & =\frac{\partial F_{1}}{\partial q_{i}}\left(q, q_{0}, t\right), \\
p_{0_{i}} & =-\frac{\partial F_{1}}{\partial q_{0_{i}}}\left(q, q_{0}, t\right), \\
0 & =H\left(q, \frac{\partial F_{1}}{\partial q}, t\right)+\frac{\partial F_{1}}{\partial t} .
\end{aligned}
$$

Equation (8) is known as the Hamilton-Jacobi equation and allows us to solve for the generating function $F_{1}$, whereas equations (6) and (7) solve the boundary value problem that consists in going from $q_{0}$ to $q$ in $t$ units of time.

Now let us consider more general generating functions. Let $\left(i_{1}, \cdots, i_{p}\right)\left(i_{p+1}, \cdots, i_{n}\right)$ and $\left(k_{1}, \cdots, k_{r}\right)$ $\left(k_{r+1}, \cdots, k_{n}\right)$ be two partitions of the set $(1, \cdots, n)$ into two non-intersecting parts such that $i_{1}<\cdots<i_{p}$,

\footnotetext{
${ }^{a}$ as mentioned in the introduction the improved CW equations are the CW equations that take into account the $J_{2}$ gravity coefficient.

${ }^{\mathrm{b}}$ Consider for instance the converging Taylor series of $(1-t)^{x}$ with respect to $x$. As $t$ goes to 1 , first terms are no more dominant.
} 
$i_{p+1}<\cdots<i_{n}, k_{1}<\cdots<k_{r}$ and $k_{r+1}<\cdots<k_{n}$ and define $I_{p}=\left(i_{1}, \cdots, i_{p}\right), \bar{I}_{p}=\left(i_{p+1}, \cdots, i_{n}\right)$, $K_{r}=\left(k_{1}, \cdots, k_{r}\right)$ and $\bar{K}_{r}=\left(k_{r+1}, \cdots, k_{n}\right)$. The generating function

$$
F_{I_{p}, K_{r}}\left(q_{I_{p}}, p_{\bar{I}_{p}}, q_{0_{K_{r}}}, p_{0_{\bar{K}_{r}}}, t\right)=F\left(q_{i_{1}}, \cdots, q_{i_{p}}, p_{i_{p+1}}, \cdots, p_{i_{n}}, q_{0_{k_{1}}}, \cdots, q_{0_{k_{r}}}, p_{0_{k_{r+1}}}, \cdots, p_{0_{k_{n}}}, t\right)
$$

verifies:

$$
\begin{aligned}
p_{I_{p}} & =\frac{\partial F_{I_{p}, K_{r}}}{\partial q_{I_{p}}}\left(q_{I_{p}}, p_{\bar{I}_{p}}, q_{0_{K_{r}}}, p_{0_{\bar{K}_{r}}}, t\right), \\
q_{\bar{I}_{p}} & =-\frac{\partial F_{I_{p}, K_{r}}}{\partial q_{\bar{I}_{p}}}\left(q_{I_{p}}, p_{\bar{I}_{p}}, q_{0_{K_{r}}}, p_{0_{\bar{K}_{r}}}, t\right), \\
p_{0_{K_{r}}} & =-\frac{\partial F_{I_{p}, K_{r}}}{\partial q_{0_{K_{r}}}}\left(q_{I_{p}}, p_{\bar{I}_{p}}, q_{0_{K_{r}}}, p_{0_{\bar{K}_{r}}}, t\right), \\
q_{\bar{K}_{r}} & =\frac{\partial F_{I_{p}, K_{r}}}{\partial p_{0 \bar{K}_{r}}}\left(q_{I_{p}}, p_{\bar{I}_{p}}, q_{0_{K_{r}}}, p_{0_{\bar{K}_{r}}}, t\right), \\
0 & =H\left(q_{I_{p}},-\frac{\partial F_{I_{p}, K_{r}}}{\partial p_{\bar{I}_{p}}}, \frac{\partial F_{I_{p}, K_{r}}}{\partial q_{I_{p}}}, p_{\bar{I}_{p}}, t\right)+\frac{\partial F_{I_{p}, K_{r}}}{\partial t} .
\end{aligned}
$$

Equation (14) is the general form of the Hamilton-Jacobi equation and allows one to solve for the generating function $F_{I_{p}, K_{r}}$. On the other hand, Eqs. (10), (11), (12) and (13) solve the boundary value problem that consists in going from $\left(q_{I_{p}}, p_{\bar{I}_{p}}\right)$ to $\left(q_{0_{K_{r}}}, p_{0_{\bar{K}_{r}}}\right)$ in $t$ units of time. Among the $4^{n}$ generating functions defined above, we can recover the 4 classical kinds of generating functions discussed by Goldstein; ${ }^{26}$ if the partitions are $(1, \cdots, n)()$ and ()$(1, \cdots, n)$ (i.e., $p=n$ and $r=0)$ we recover the generating function $F_{2}$. The case $p=0$ and $r=n$ corresponds to the generating function $F_{3}$ and if $p=0$ and $r=0$ we obtain $F_{4}$.

If the Hamiltonian in Eq. (14) is $H^{h}$, as defined by Eq. (5), then the generating functions are associated with the phase flow that describes the relative motion and they solve relative boundary value problems. In terms of notation, $\left(q_{0}, p_{0}, q, p\right)$ becomes $\left(\Delta q_{0}, \Delta p_{0}, \Delta q, \Delta p\right)$.

\section{The numerics of the algorithm}

There are two methods for finding the generating functions, one can either solve the Hamilton-Jacobi equation (Eq. (14)) or use an indirect approach based on the initial value problem. These methods are detailed in $^{22}$ and in the following we briefly review their characteristics. They both have their advantages and drawbacks and one usually needs to combine both of them. We will discuss this issue at the end of this section.

\section{Solving the Hamilton-Jacobi equation}

We assume the generating functions can be expressed as a Taylor series about the reference trajectory in its spatial variables.

$$
F_{I_{p}, K_{r}}(y, t)=\sum_{q=2}^{\infty} \sum_{\substack{i_{1}, \cdots, i_{2 n}=0 \\ i_{1}+\cdots+i_{2 n}=q}}^{q} \frac{1}{i_{1} ! \cdots i_{2 n} !} f_{i_{1}, \cdots, i_{2 n}}^{p, r}(t) y_{1}^{i_{1}} \cdots y_{2 n}^{i_{2 n}},
$$

where $y=\left(\Delta q_{I_{p}}, \Delta p_{\bar{I}_{p}}, \Delta q_{0_{K_{r}}}, \Delta p_{0_{\bar{K}_{r}}}\right)$. We substitute this expression in Eq. (14), where $H$ is the Hamiltonian for the relative motion (Eq. 5). The resulting equation is an ordinary differential equation that has the following structure:

$$
P\left(y, f_{i_{1}, \cdots, i_{2 n}}^{p, r}(t), \dot{f}_{i_{1}, \cdots, i_{2 n}}^{p, r}(t)\right)=0,
$$

where $P$ is a series in $y$ with time dependent coefficients that are functions of $f_{i_{1}, \cdots, i_{2 n}}^{p, r}(t), \dot{f}_{i_{1}, \cdots, i_{2 n}}^{p, r}(t)$. Equation (16) holds for all $y$ if and only if all the coefficients of $P$ are zero. In this manner, we transform the ordinary differential equation (16) into a set of ordinary differential equations whose solutions are the coefficients of the generating function $F_{I_{p}, K_{r}}$. Now suppose that we have knowledge of the generating functions up to order $N-1$, then from Eq. (15) we deduce that we need to solve $\frac{(N+5) !}{N ! 5 !}$ additional ordinary differential equations to find order $N$. 
This approach provides us with a closed form approximation of the generating functions. However, there are inherent difficulties as generating functions may develop singularities which prevent the integration from going further (see ${ }^{18,27,28}$ for more details on singularities). Techniques have been developed ${ }^{22}$ to bypass this problem but have a cost in terms of computation. Typically, this method should only be used to solve generating functions over a short period of time.

\section{The indirect approach}

By definition, generating functions implicitly define the canonical transformation they are associated with. Hence, we may compute the generating functions from the canonical transformation, that is, compute the generating functions for the phase flow transformation from knowledge of the phase flow. In this section, we develop an algorithm based on these ideas (more details may be gleaned in ${ }^{22}$ ).

Recall Hamilton's equations of motion for the relative motion:

$$
\left(\begin{array}{c}
\dot{\Delta} q \\
\dot{\Delta p}
\end{array}\right)=\left(\begin{array}{cc}
0 & I_{n} \\
-I_{n} & 0
\end{array}\right) \nabla H^{h}(\Delta q, \Delta p, t) .
$$

We suppose that $\Delta q\left(\Delta q_{0}, \Delta p_{0}, t\right)$ and $\Delta p\left(\Delta q_{0}, \Delta p_{0}, t\right)$ can be expressed as series in the initial conditions $\left(\Delta q_{0}, \Delta p_{0}\right)$ with time dependent coefficients. We truncate the series to order $N$ and insert these into Eq. (17). Hamilton's equations reduce to a series in $\left(\Delta q_{0}, \Delta p_{0}\right)$ whose coefficients depend on the time dependent coefficients and the derivatives of the series $\Delta q\left(\Delta q_{0}, \Delta p_{0}, t\right)$ and $\Delta p\left(\Delta q_{0}, \Delta p_{0}, t\right)$. As in the previous section, we balance terms of the same order and transform Hamilton's equations into a set of ordinary differential equations whose variables are the time dependent coefficients defining $\Delta q$ and $\Delta p$ as a series in $\Delta q_{0}$ and $\Delta p_{0}$. Using $\Delta q\left(\Delta q_{0}, \Delta p_{0}, t_{0}\right)=\Delta q_{0}$ and $\Delta p\left(\Delta q_{0}, \Delta p_{0}, t_{0}\right)=\Delta p_{0}$ as initial conditions for the integration, we are able to compute an approximation of order $N$ for the phase flow. At linear order, this approach recovers the state transition matrix. Then, a series inversion of the phase flow provides us with the gradient of the generating functions that can be integrated to find the generating functions.

The main advantage of this approach is that the phase flow is never singular, therefore the system of ordinary differential equations are always well-defined. However, this method requires us to solve more equations than the previous method ${ }^{c}$ and provides us with the expression of the generating function at a given time only (the time at which we perform the series inversion). In addition a symplectic algorithm should be used to integrate the ordinary differential equations, otherwise we obtain an inconsistent expression of the gradient of the generating functions that cannot be integrated (some exactness conditions are not satisfied ${ }^{29}$ ).

In this paper, we combine both methods, we first solve the initial value problem over a long time span using the symplectic implicit Runge-Kutta integrator built in Mathematica $^{\circledR}$. Then we compute the generating functions at a time of interest, say $t_{1}$, and solve the Hamilton-Jacobi equation about $t_{1}$, with initial conditions equal to the values of the generating functions at $t_{1}$ found using the indirect approach. For solving the Hamilton-Jacobi equation, we use the Mathematica ${ }^{\circledR}$ built in function NDSolve with its default attributes ${ }^{\mathrm{d}}$.

\section{Formation design}

In the previous sections we introduced a dynamical model, defined a reference trajectory and presented an algorithm whose outputs are the generating functions associated with the phase flow describing the relative motion. We have also explained how these generating functions may be used to solve two-point boundary value problems. We now combine all the above and use it to design spacecraft formations. We first use the "combined" algorithm to find the generating function $F_{1}$ up to order 4, that is we need to solve 203 ordinary differential equations (see appendix for computational times). Once the generating functions are known, we can solve any position to position boundary value problem with only six polynomial evaluations (Eqs. (6) and (7)).

\footnotetext{
' If we want to find the generating function up to order $N$, then we need to solve $6 \sum_{k=1}^{N-1} \frac{(k+5) !}{k ! 5 !}$ equations, which is always greater than $\sum_{k=2}^{N} \frac{(k+5) !}{k ! 5 !}$, the number of equations that need to be solved using the direct approach.

$\mathrm{d} N D$ Solve switches between a non-stiff Adams method and a stiff Gear method and achieves a precision of $10^{-10}$.
} 


\section{A. Multi-task mission}

We consider 4 imaging satellites flying in formation about the reference trajectory. We want to plan spacecraft maneuvers over the next month knowing that they must observe the Earth, i.e., must be in a given configuration $C_{i}$ at the following instants (chosen arbitrarily for our study):

$$
\begin{array}{cc}
t_{0}=0, & t_{1}=5 \text { days } 22 \text { hours }, \quad t_{2}=10 \text { days } 20 \text { hours }, \\
t_{3}=16 \text { days } 2 \text { hours }, & t_{4}=21 \text { days } 14 \text { hours }, \quad t_{5}=26 \text { days } 20 \text { hours } .
\end{array}
$$

Define the local horizontal by the unit vectors $\left(\hat{e}_{1}, \hat{e}_{2}\right)$ such that $\hat{e}_{2}$ is along $r^{0} \times v^{0}$ and $\hat{e}_{1}$ is along $\hat{e}_{2} \times r^{0}$. At every $t_{i}$, the configuration $C_{i}$ is defined by the four following relative positions (or slots):

$$
q^{1}=700 m \hat{e}_{1}, \quad q^{2}=-700 m \hat{e}_{1}, \quad q^{3}=700 m \hat{e}_{2}, \quad q^{4}=-700 m \hat{e}_{2} .
$$

Note that at $t_{i}, q^{1}$ is in front of the reference state (in the local horizontal plane), $q^{2}$ is behind, $q^{3}$ is on the left and $q^{4}$ is on the right (see figure 1(a)). At each $t_{i}$, there must be one spacecraft per slot and we want to determine the sequence of reconfigurations that minimizes the total fuel expenditure ${ }^{\mathrm{e}}$ (other cost functions such as equal fuel consumption for each spacecraft may be considered as well). For the first mission, there are 4 ! configurations (number of permutation of the set $\{1,2,3,4\}$ ). For the second mission, for each of the previous 4 ! configurations, there are again 4 ! configurations, that is a total of $4 !^{2}$ possibilities. Thus for 5 missions there are $4 !^{5}=7,962,624$ possible configurations.

In this paper, we focus on impulsive controls, but the method we develop can equivalently apply to continuous thrust problems. Indeed, continuous thrust problems are usually solved using optimal control theory and reduce to a set of necessary conditions that are formulated as a Hamiltonian two-point boundary value problem. This boundary value problem can in turn be solved using the method we present in this paper. $^{24}$ Let us now design the above mission. We assume impulsive controls that consist of impulsive thrusts applied at $t_{i \in[0,5]}$. For each of the four spacecraft, we need to compute the velocity at $t_{i}$ so that the spacecraft moves to its position specified at $t_{i+1}$ under gravitational forces only. As a result, we must solve $5 \cdot 4 !=120$ position to position boundary value problems (given two positions at $t_{i}$ and $t_{i+1}$, we need to compute the associated velocity). Using the generating functions, this problem can be handled at the cost of only 720 function evaluations (computation times are given in the appendix). Then, we need to evaluate the fuel expenditure (sum of the norm of all the required impulses, assuming zero relative velocities at the initial and final times) for all the permutations (there are 7,962,624 combinations) to find the sequence that minimizes the cost function. Figure 4 represents the number of configurations as a function of the values of the cost function. We notice that most of the configurations require at least three times more fuel than the best configuration, and less than $6 \%$ yield values of the cost function that are less than twice the value associated with the best configuration. The cost function for the optimal sequence of reconfigurations is $0.00644 \mathrm{~km} \cdot \mathrm{s}^{-1}$ whereas it is $0.0396 \mathrm{~km} \cdot \mathrm{s}^{-1}$ in the least optimal design. In the optimal case, the four spacecraft have the following positions:

Spacecraft 1: $\quad\left(t_{0}, q^{1}\right), \quad\left(t_{1}, q^{2}\right), \quad\left(t_{2}, q^{2}\right), \quad\left(t_{3}, q^{2}\right), \quad\left(t_{4}, q^{2}\right), \quad\left(t_{5}, q^{2}\right)$. Spacecraft 2: $\quad\left(t_{0}, q^{2}\right), \quad\left(t_{1}, q^{1}\right), \quad\left(t_{2}, q^{1}\right), \quad\left(t_{3}, q^{1}\right), \quad\left(t_{4}, q^{1}\right), \quad\left(t_{5}, q^{1}\right)$. Spacecraft 3: $\quad\left(t_{0}, q^{3}\right), \quad\left(t_{1}, q^{4}\right), \quad\left(t_{2}, q^{4}\right) \quad\left(t_{3}, q^{4}\right), \quad\left(t_{4}, q^{3}\right), \quad\left(t_{5}, q^{4}\right)$. Spacecraft 4: $\quad\left(t_{0}, q^{4}\right), \quad\left(t_{1}, q^{3}\right), \quad\left(t_{2}, q^{3}\right) \quad\left(t_{3}, q^{3}\right), \quad\left(t_{4}, q^{4}\right), \quad\left(t_{5}, q^{3}\right)$.

whereas the worst scenario corresponds to:

Spacecraft 1: $\quad\left(t_{0}, q^{1}\right), \quad\left(t_{1}, q^{1}\right), \quad\left(t_{2}, q^{2}\right), \quad\left(t_{3}, q^{2}\right), \quad\left(t_{4}, q^{1}\right), \quad\left(t_{5}, q^{2}\right)$. Spacecraft 2: $\quad\left(t_{0}, q^{2}\right), \quad\left(t_{1}, q^{2}\right), \quad\left(t_{2}, q^{3}\right), \quad\left(t_{3}, q^{4}\right), \quad\left(t_{4}, q^{4}\right), \quad\left(t_{5}, q^{3}\right)$. Spacecraft 3: $\quad\left(t_{0}, q^{3}\right), \quad\left(t_{1}, q^{3}\right), \quad\left(t_{2}, q^{1}\right) \quad\left(t_{3}, q^{3}\right), \quad\left(t_{4}, q^{3}\right), \quad\left(t_{5}, q^{4}\right)$. Spacecraft 4: $\quad\left(t_{0}, q^{4}\right), \quad\left(t_{1}, q^{4}\right), \quad\left(t_{2}, q^{4}\right) \quad\left(t_{3}, q^{1}\right), \quad\left(t_{4}, q^{2}\right), \quad\left(t_{5}, q^{1}\right)$.

We may verify, a posteriori, if the solutions found meet the mission goals, i.e., if the order 4 approximation of the dynamics is sufficient to simulate the true dynamics. Explicitly comparing the analytical solution with numerically integrated results shows that the spacecraft are at the desired positions at every $t_{i}$ with a maximum error of $1.5 \cdot 10^{-8} \mathrm{~km}$.

${ }^{\text {e}}$ Each impulses instantaneously change the velocity vector. The norm of this change quantifies the fuel expenditure 


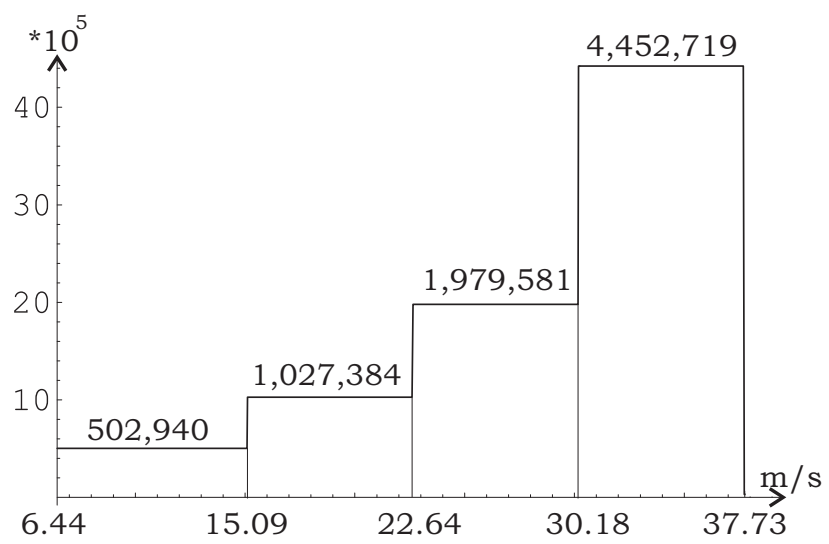

Figure 4. Number of configurations as a function of the value of the cost function

\section{Considerations on collision management}

Our algorithm does not consider the risk of collision in the design. However, it provides a simple way to check afterwards if there is collision. Recall the indirect method, it is based on the initial value problem and essentially consists in solving Hamilton's equations for an approximation of the flow. Once such a solution is found, we can generate any trajectory at the cost of a function evaluation, there is no need to integrate Hamilton's equations again. Checking for collisions is again a combinatorial problem and therefore our approach is particularly adapted to this. As an example let us verify if the design we proposed for the multi-task mission yields collisions. In figure 5 we plot the distance between each of the spacecraft. We remark that only spacecraft 1 and 2, and spacecraft 3 and 4 may collide (relative distance less than $100 \mathrm{~m}$ ). A detail of the figure shows that spacecraft 1 and 2 get as close as 40 meters after about 80 hours and spacecraft 3 and 4 may eventually collide.

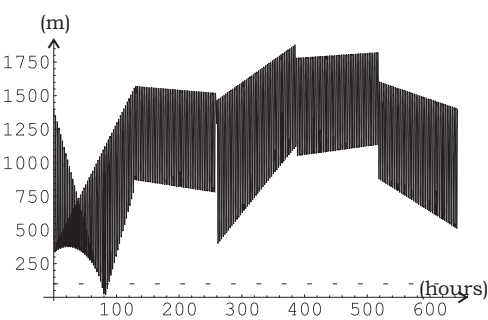

(a) Distance between Spacecraft 1 and (b) 2

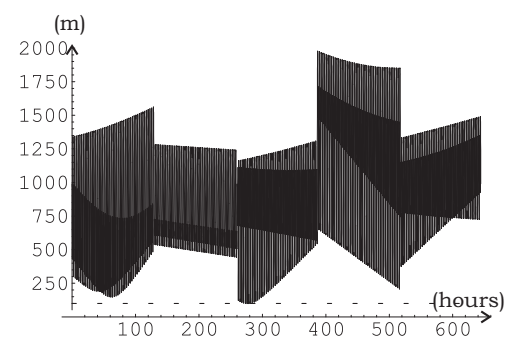

(d) Distance between Spacecraft 2 and

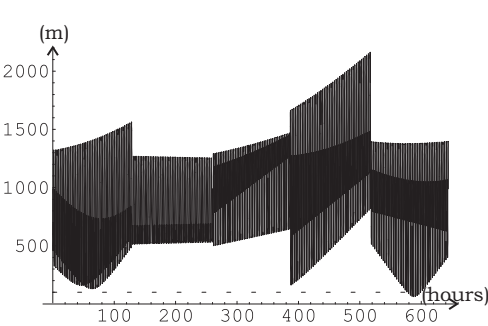

(b)

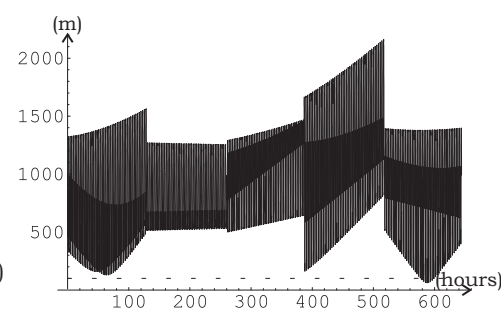

3

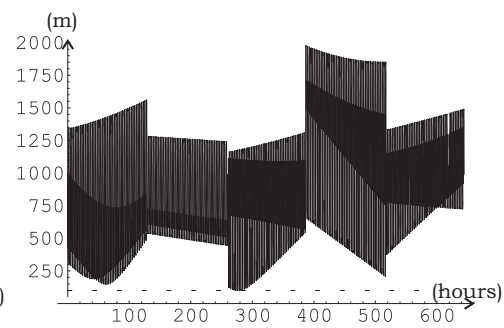

(c) Distance between Spacecraft 1 and 4

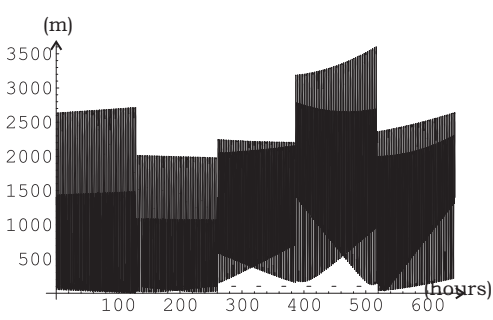

(f) Distance between Spacecraft 3 and 4

Figure 5. Distance between the spacecraft as a function of time

It can be proven that for this specific mission, there is no design that prevents the relative motion of the spacecraft to be less than $100 \mathrm{~m}$. In the best scenario, the smallest relative distance between the spacecraft 
is about $15 \mathrm{~m}$, and is achieved in 3,360 different designs. Among these 3,360 possibilities, we represent in figure 6 the time history of the relative distance between the spacecraft for the design that achieves minimum fuel expenditure (the total fuel expenditure is $60 \%$ larger than in the best case). This scenario corresponds to:

Spacecraft 1: $\quad\left(t_{0}, q^{1}\right), \quad\left(t_{1}, q^{2}\right), \quad\left(t_{2}, q^{3}\right), \quad\left(t_{3}, q^{3}\right), \quad\left(t_{4}, q^{4}\right), \quad\left(t_{5}, q^{3}\right)$.

Spacecraft 2: $\quad\left(t_{0}, q^{2}\right), \quad\left(t_{1}, q^{3}\right), \quad\left(t_{2}, q^{4}\right), \quad\left(t_{3}, q^{4}\right), \quad\left(t_{4}, q^{3}\right), \quad\left(t_{5}, q^{4}\right)$.

Spacecraft 3: $\quad\left(t_{0}, q^{3}\right), \quad\left(t_{1}, q^{4}\right), \quad\left(t_{2}, q^{1}\right) \quad\left(t_{3}, q^{2}\right), \quad\left(t_{4}, q^{1}\right), \quad\left(t_{5}, q^{2}\right)$.

Spacecraft 4: $\quad\left(t_{0}, q^{4}\right), \quad\left(t_{1}, q^{1}\right), \quad\left(t_{2}, q^{2}\right) \quad\left(t_{3}, q^{1}\right), \quad\left(t_{4}, q^{2}\right), \quad\left(t_{5}, q^{1}\right)$.
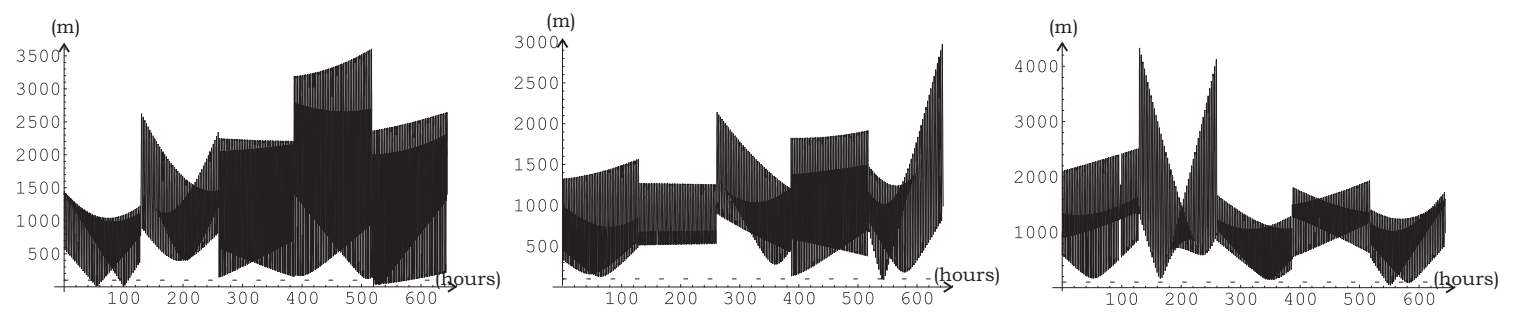

(a) Distance between Spacecraft 1 and (b)

2

(b) Distance between Spacecraft 1 and
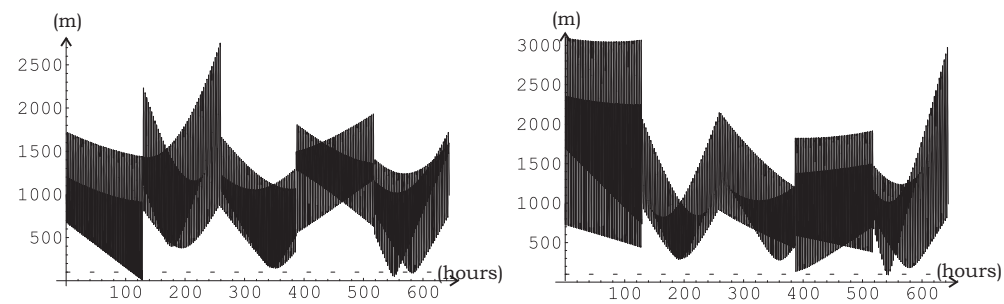

4

(d) Distance between Spacecraft 2 and (e) Distance between Spacecraft 2 and 3

3

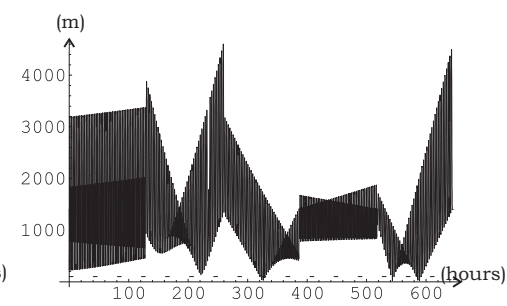

(f) Distance between Spacecraft 3 and

Figure 6. Distance between the spacecraft as a function of time

For times at which the spacecraft are close to each other, we may use some local control laws to perform small maneuvers to ensure appropriate separation.

Another option consists of changing the configurations at $t_{i}$ so that there exists a sequence of reconfigurations such that the relative distance between the spacecraft stay larger than $100 \mathrm{~m}$. This can easily be done using our approach since $F_{1}$ is already known. Solving a new design would only require 720 function evaluations.

In the above example we take advantage of our algorithm to perform the required design, that is, we are able to plan missions involving several spacecraft over a month using non-trivial dynamics while minimizing a given cost function. Such a design is possible because we focus directly on specifying the problem as a series of boundary value problems. Solution of this problem using a more traditional approach to solving boundary value problems would have required direct integration of the equations of motion for each of the 720 boundary value problems.

However, we have not taken full advantage of our algorithm yet, as the above example does not provide insight on the dynamics. We now consider a different mission to remedy this and show how our algorithm may be used for analytical studies.

\section{B. A different multi-task mission}

For simplicity, we assume that the spacecraft must achieve only one task, that is we constrain the geometry of the formation at $t_{0}$ and $t_{1}$. However, instead of imposing absolute relative positions, we only require the spacecraft to be equally spaced on a circle of a given radius in the local horizontal plane at $t_{1}$. Such a constraint is more realistic, especially for imaging satellites as rotations of the formation about the local vertical should not influence performance. In this problem, combinatorics and smooth functional analysis 
are mixed together. Indeed, the positions of the four slots are given by a variable $\theta$ ( $\theta$ indicates the position of the first slot, the other slots are determined from the constraint that they should be equally spaced). Then, we need to solve a combinatorial problem as in the previous case. To find the $\theta$ that minimizes the cost function, we use the polynomial approximation of the generating functions provided by our algorithm to express the cost function as a one dimensional polynomial in $\theta$. Variations of the cost function are determined analytically by computing the derivative of the cost function.

We choose the initial position to be as in the previous example and require the spacecraft to be equally spaced at $t_{1}$ on a circle of radius 700 meters in the local horizontal plane. In addition, we assume zero relative velocities at the initial and final times and again choose the cost function to be the sum of the norm of the required impulses. As before, $\left(\hat{e}_{1}, \hat{e}_{2}\right)$ span the local horizontal plane and we define $\theta$ as the angle between the relative position vector and $\hat{e}_{1}$. Since $\theta$ is allowed to vary from 0 to $2 \pi$ (i.e., slot 1 describes the whole circle as $\theta$ goes from 0 to $2 \pi$ ), we may consider that spacecraft 1 always goes from slot 1 to slot 1. As a consequence, there are 3 ! free configurations. In figure 7 , we plot the values of the cost function as a function of $\theta$ for each of the configurations. The best design is the one for which $\theta=3.118 \mathrm{rad}$, spacecraft 1 goes from slot 1 to slot 1 , spacecraft 2 from 2 to 3 , spacecraft 3 from 3 to 2 and spacecraft 4 from 4 to 4 .

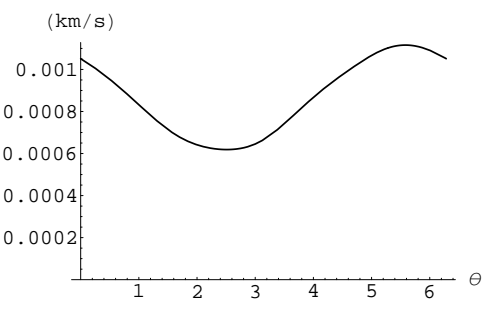

(a) Spacecraft goes from slots $(1,2,3,4)$ to $(1,2,3,4)$

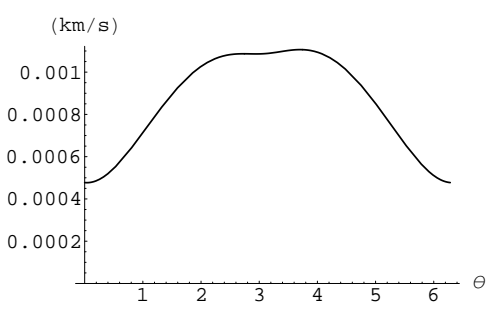

(d) Spacecraft goes from slots $(1,2,3,4)$ to $(1,3,4,2)$

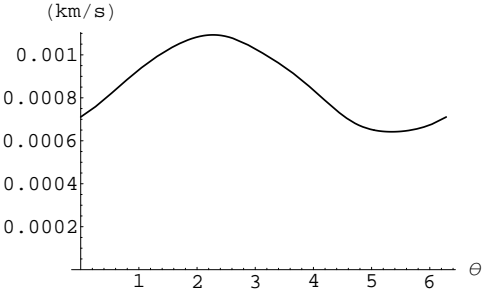

(b) Spacecraft goes from slots $(1,2,3,4)$ to $(1,2,4,3)$

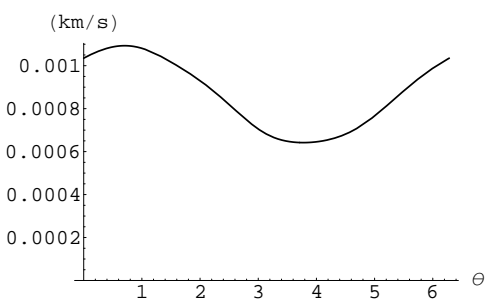

(e) Spacecraft goes from slots $(1,2,3,4)$ to $(1,4,2,3)$

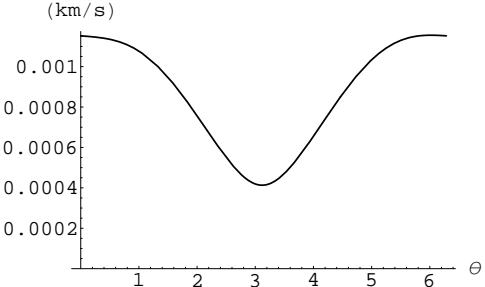

(c) Spacecraft goes from slots $(1,2,3,4)$ to $(1,3,2,4)$

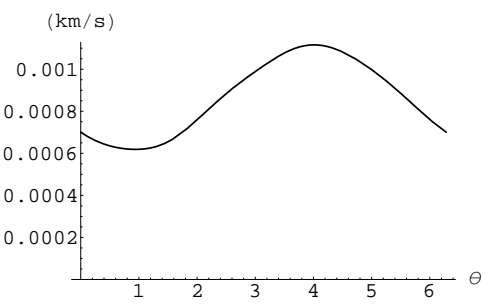

(f) Spacecraft goes from slots $(1,2,3,4)$ to $(1,4,3,2)$

Figure 7. Cost function as a function of $\theta$ for each configuration

If several missions need to be planned, then a new variable is introduced for each and a multi-variable polynomial must be studied. As a result, minima of the cost function are found by evaluating as many derivatives as there are missions.

Through this example, we have gained insight on the dynamics by using the analytical approximation of the generating function and were able to solve the fuel optimal reconfiguration problem. The method we use is very general and can be applied to solve any reconfiguration problem given that the constraints on the configurations are holonomic.

\section{Stable trajectories}

Now we focus on another crucial, but difficult, design issue for spacecraft formations. We search for configurations, called "stable" configurations, such that the spacecraft stay close to the reference trajectory over a long time span.

\section{Definitions}

Let us first define the notion of stable formation more precisely. Let $T$ be a given instant and $M$ a real number. 
Definition IV.1 (Stable relative trajectory). A relative trajectory between two spacecraft is $(M, T)$ stable if and only if their relative distance never exceeds $M$ over the time span $[0, T]$.

Definition IV.2 (Stable formation). A formation of spacecraft is $(M, T)$-stable if and only if all the spacecraft have $(M, T)$-stable relative trajectories with respect to the reference trajectory.

Periodic formations are instances of stable formations, they are $(M, \infty)$-stable. We also point out that our definition recovers the notion of Lyapunov stability: $(M, \infty)$-stable relative trajectories are stable in the sense of Lyapunov. In this paper, we focus on $(M, T)$-stable formations with $T$ large but finite, the approach we present is not appropriate to find $(M, \infty)$-stable configurations. However, when the reference trajectory is periodic Guibout and Scheeres ${ }^{23}$ developed a technique based on generating functions and Hamilton-Jacobi theory to find periodic configurations.

\section{Stable trajectories as solutions to two-point boundary value problems}

In order to use the theory we have presented above, we formulate the search for stable trajectories as two-point boundary value problems.

Define the local vertical plane as the two-dimensional vector space perpendicular to the velocity vector of the reference trajectory. In other words, the local vertical is spanned by $\left(\hat{f}_{1}, \hat{f}_{2}\right)$ where $\hat{f}_{1}$ and $\hat{f}_{2}$ are two unit vectors along $r^{0} \times v^{0}$ and $v^{0} \times \hat{f}_{1}$ respectively. In the local vertical plane, we use polar coordinates, $\left(r-r^{0}, \theta\right), \theta$ being the angle between $\hat{f}_{1}$ and the local relative position vector $r-r^{0}$. We denote by $\mathcal{C}_{t}^{r}$ the circle of radius $r$ centered on the reference trajectory that lies in the local vertical plane at $t$. A position on this circle is fully determined by $\theta$ (see figure 8). Then given instant $t_{f}>t_{0}$ and a distance $r_{f}>0$, the circle $\mathcal{C}_{t_{f}}^{r_{f}}$ is defined.

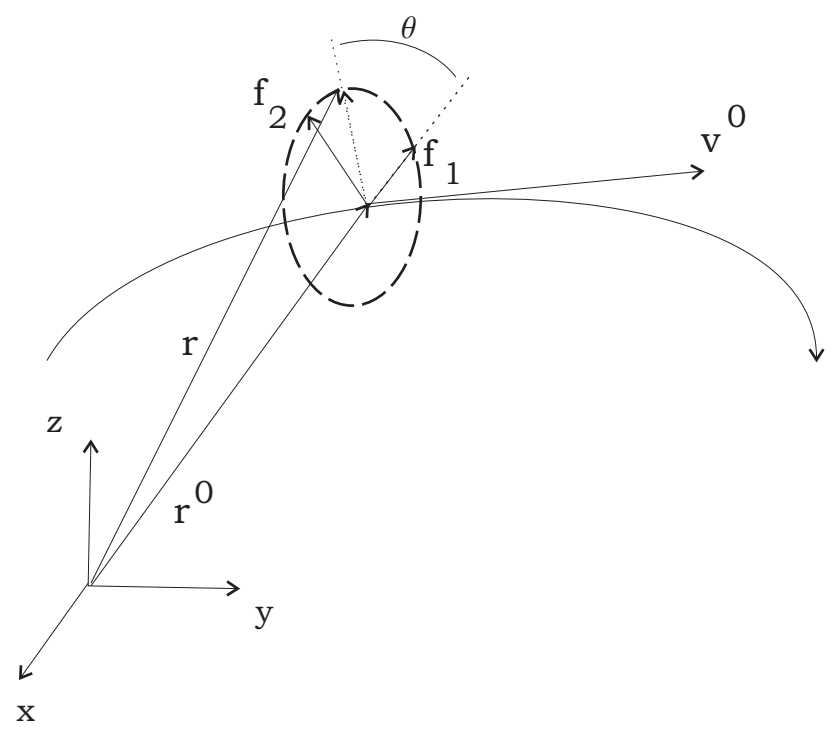

Figure 8. Representation of the local geometry

Before searching for stable configurations, we first introduce a new methodology to find $(M, T)$-stable relative trajectories for a single spacecraft about the reference trajectory defined above. Consider the following two-point boundary value problem:

Find all trajectories going from the initial position of the spacecraft to any point on $\mathcal{C}_{t_{f}}^{r_{f}}$ in $t_{f}-t_{0}$ units of time where $r_{f}<M$ (figure 9).

Solutions to this boundary value problem have the following properties:

1. they contain $(M, T)$-stable relative trajectories.

2. they contain relative trajectories that are not $(M, T)$-stable, i.e., trajectories that go far from the reference trajectory in the time interval $\left(0, t_{f}\right)$ but come back close to the reference trajectory at $t_{f}$. We point out that many of these trajectories are ignored by our algorithm since it uses a local approximation of the dynamics. 


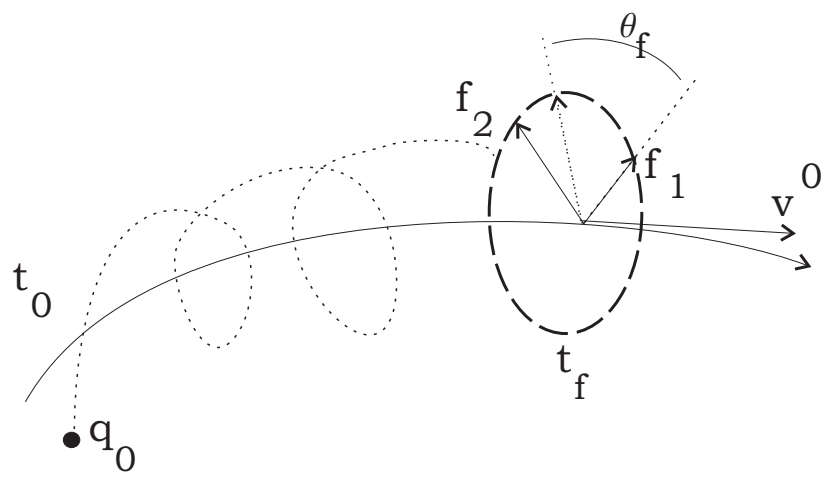

Figure 9. Boundary value problem

On the other hand, we know that stable trajectories must have similar orbit elements as compared to the reference trajectory. Therefore, to discriminate between the solutions to the two-point boundary value problem we can use orbit elements, especially since we know, a priori, that the longitude of the ascending node and the argument of perigee have secular drifts. This leads us to define a cost function $J$ as:

$$
J=\frac{1}{4}\left\|\Delta \omega_{t_{f}}\right\|+\frac{1}{4}\left\|\Delta \omega_{t_{f}}-\Delta \omega_{t_{0}}\right\|+\frac{1}{4}\left\|\Delta \Omega_{t_{f}}\right\|+\frac{1}{4}\left\|\Delta \Omega_{t_{f}}-\Delta \Omega_{t_{0}}\right\|,
$$

where $\left\|\Delta \omega_{t_{f}}\right\|$ corresponds to the relative argument of perigee at $t_{f}$, i.e, the difference at $t_{f}$ between the argument of perigee of the spacecraft trajectory and the argument of perigee of the reference trajectory, $\left\|\Delta \omega_{t_{f}}-\Delta \omega_{t_{0}}\right\|$ characterizes the change in the relative argument of perigee between $t_{0}$ and $t_{f}$ and the other terms are similar and involve the longitude of the ascending node instead.

Let us now consider the following boundary value problem: Find all trajectories going from the initial position of the spacecraft to any point on $\mathcal{C}_{t_{f}}^{r_{f}}$ in $t_{f}-t_{0}$ units of time that minimize $J$.

From the above discussion, we conclude that solutions to this boundary value problem characterize stable relative trajectories.

\section{Methodology}

We showed in the previous section that the search for stable trajectories reduces to solving a two-point boundary value problem while minimizing a given cost function. In this section, we solve this problem using the generating function theory introduced in the first part of this paper.

First we notice that $F_{1}$ solves the boundary value problem that consists of going from an initial position $q_{0}$ to a position $q_{f}$ in $t_{f}$ units of time. Indeed, from Eqs. (6) and (7) we have:

$$
\begin{aligned}
& p_{0}=-\frac{\partial F_{1}}{\partial q_{0}}\left(q, q_{0}, t_{f}\right), \\
& p_{f}=\frac{\partial F_{1}}{\partial q}\left(q, q_{0}, t_{f}\right) .
\end{aligned}
$$

Then we assume that $q_{f}$ describes $\mathcal{C}_{t_{f}}^{r_{f}}$, that is, $q_{f}=r_{f} \cos \left(\theta_{f}\right) \hat{f}_{1}+r_{f} \sin \left(\theta_{f}\right) \hat{f}_{2}$ where $\theta_{f}$ ranges from 0 to $2 \pi$. Since $F_{1}$ is approximated by a polynomial in $\left(q, q_{0}\right)$ with time-dependent coefficients, Eqs. (21) and (22) allow us to express $p_{0}$ and $p_{f}$ as polynomials in $\theta_{f}$ with time-dependent coefficients. Finally, with knowledge of $p_{0}\left(\theta_{f}\right), p_{f}\left(\theta_{f}\right), q_{0}$ and $q_{f}\left(\theta_{f}\right)$, we can express $J$ as a function of $\theta_{f}$ and easily find its minima $\left\{\theta_{f}^{1}, \cdots, \theta_{f}^{r}\right\}$. Stable trajectories are then those that travel from $q_{0}$ to $q_{f}=r_{f} \cos \left(\theta_{f}^{i}\right) \hat{f}_{1}+r_{f} \sin \left(\theta_{f}^{i}\right) \hat{f}_{2}, i \in[1, r]$ in $t_{f}$ units of time.

\section{Example}

Let us illustrate this procedure by searching for stable trajectories for a spacecraft whose initial position relative to the reference trajectory is $q_{0}=(495,-428.6,247.5) \mathrm{m}$ in the inertial frame or equivalently $q_{0}=$ 
$700 \cos (\pi / 4) \hat{f}_{1}+700 \sin (\pi / 4) \hat{f}_{2} \mathrm{~m}$. We use an order 4 approximation of the dynamics, $t_{f}=10 \mathrm{~d} 19 \mathrm{~h} 13 \mathrm{~min}$ and $r_{f}=700 \mathrm{~m}$. Then, using a symbolic manipulator, we express $J$ as a function of $\theta_{f}$ and plot its values in figure 10. It has two local minima at $\theta_{1}=0.671503 \mathrm{rad}$ and $\theta_{2}=2.4006615 \mathrm{rad}$ that correspond to stable trajectories. The relative motions associated with these two trajectories are represented in figures 11 and 12 over time spans smaller and larger than $t_{f}$. We notice the excellent behavior of these trajectories, they remain stable over a time interval larger than the one initially considered.

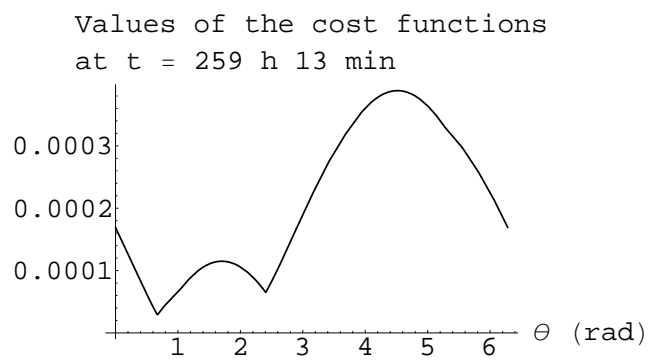

Figure 10. Cost function as a function of $\theta_{f}$

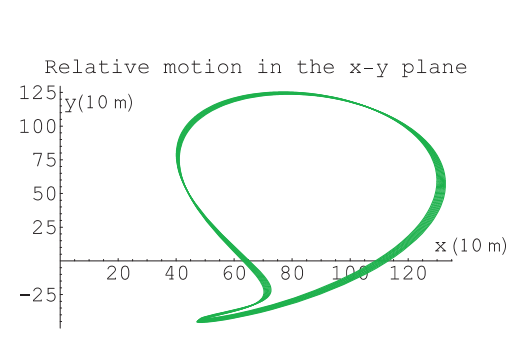

(a) $x-y$ motion during 26 hours

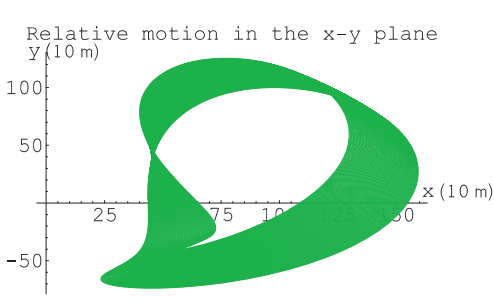

(d) $x-y \quad$ motion 11 days 19 hours

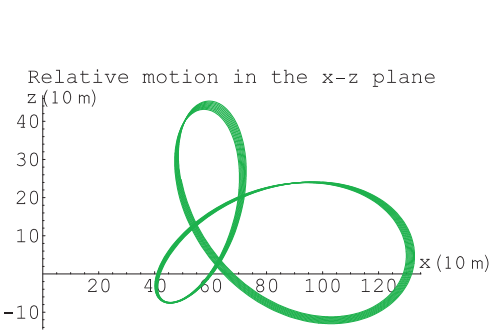

(b) $x-z$ motion during 26 hours
Relative motion in the $y-z$ plane

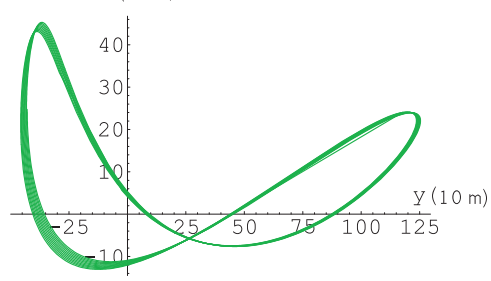

(c) $y-z$ motion during 26 hours

Relative motion in the $y-z$ plane
\[ z(10 \mathrm{~m}) \]

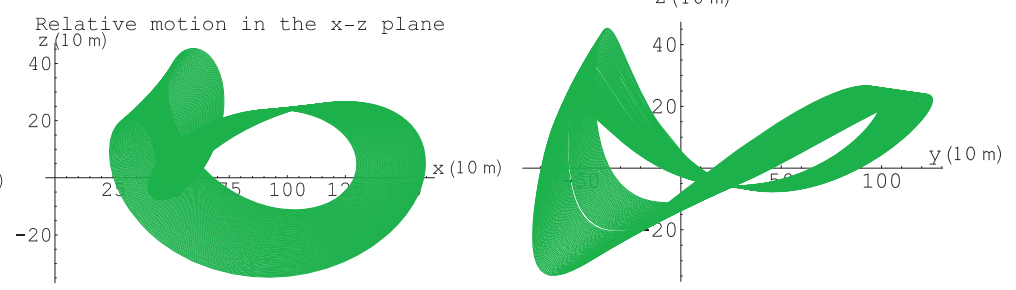

during (e) $\quad x \quad-\quad z \quad$ motion 11 days 19 hours

during (f) $y-z \quad z \quad$ motion during 11 days 19 hours

Relative motion in the $y-z$ plane
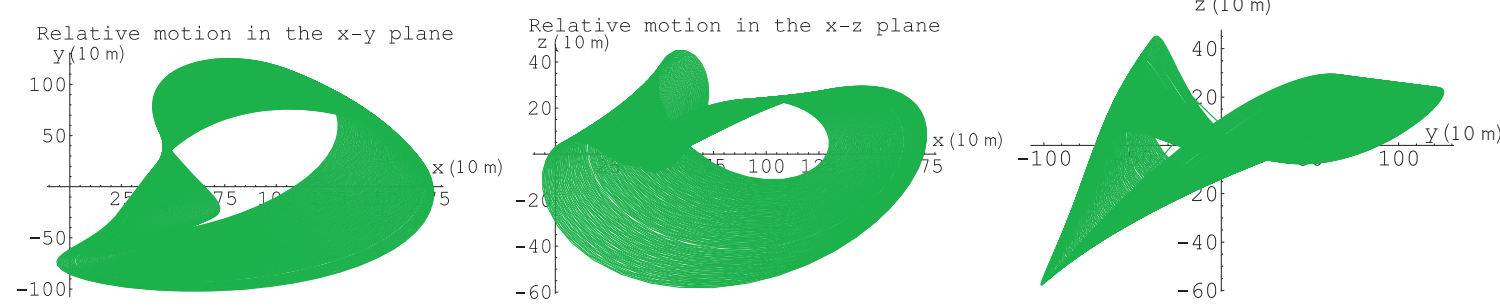

(g) $x-y \quad$ motion

21 days 11 hours

during (h) $x-z \quad$ motion

21 days 11 hours during (i) $y-z$ motion during 21 days 11 hours

Figure 11. Trajectory associated with the minimum $\theta_{f}=0.671503 \mathrm{rad}, t_{f}=10 \mathrm{~d} 19 \mathrm{~h} 13 \mathrm{~min}$. 


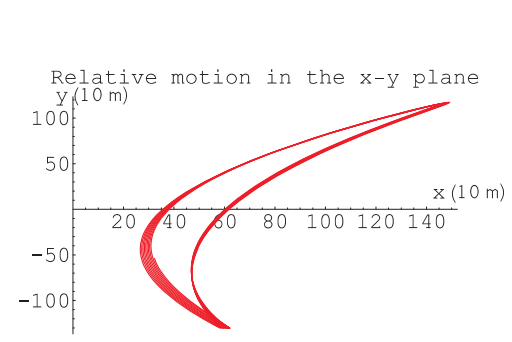

(a) $x-y$ motion during 26 hours

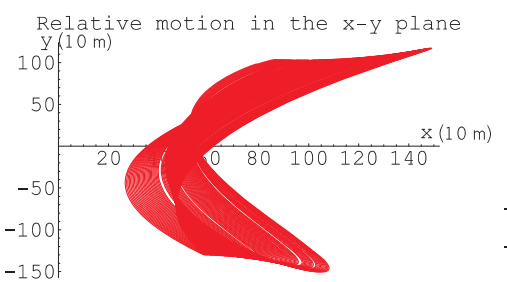

(d) $x-y \quad$ motion 11 days 19 hours

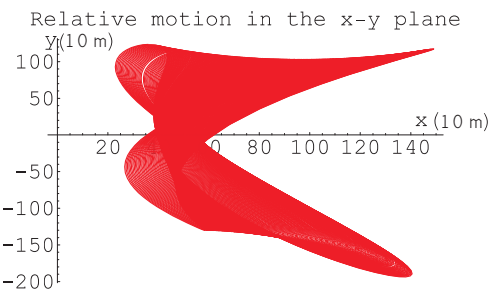

(g) $x-y \quad$ motion 21 days 11 hours

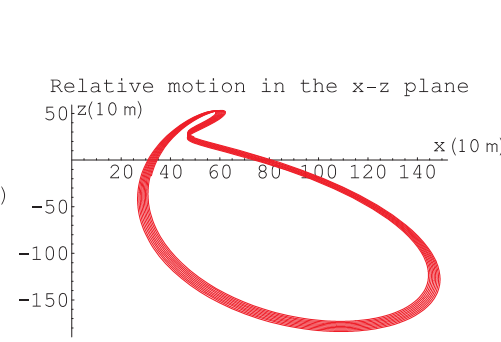

(b) $x-z$ motion during 26 hours
Relative motion in the $y-z$ plane

$\mathrm{z}(10 \mathrm{~m})$

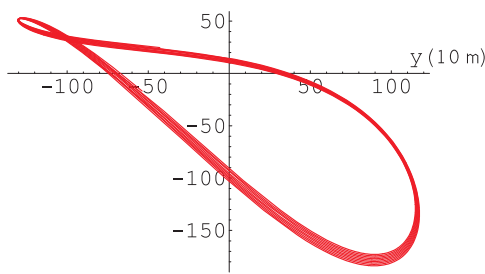

(c) $y-z$ motion during 26 hours Relative motion in the $y-z$ plane $\mathrm{z}(10 \mathrm{~m})$

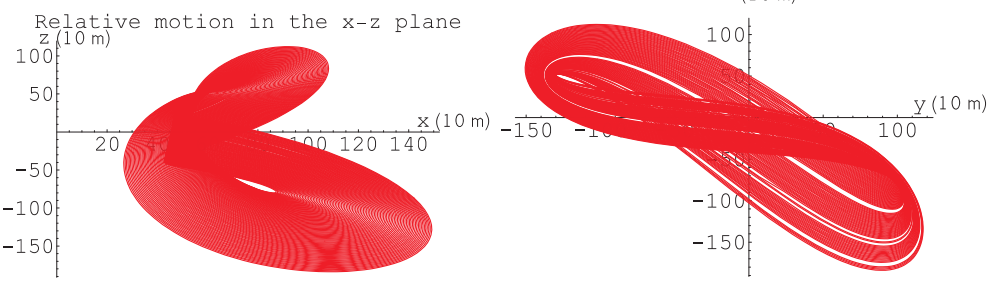

during (e) $x-z \quad z$ motion 11 days 19 hours

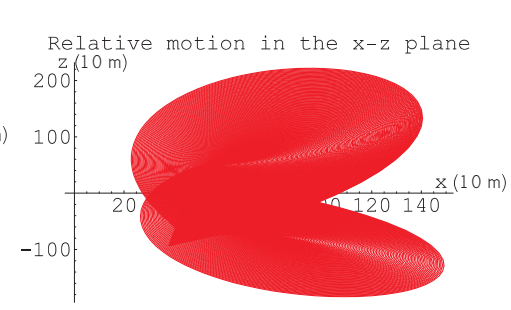
(f) $y-z$ 11 days 19 hours motion during Relative motion in the $\mathrm{y}-\mathrm{z}$ plane

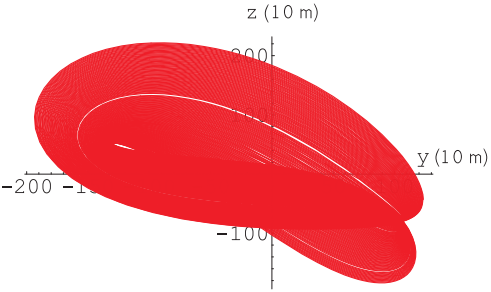
(h) $x-z$ motion during (i) $y-z$ motion during 21 days 11 hours 21 days 11 hours

Figure 12. Trajectory associated with the minimum $\theta_{f}=2.4006615 \mathrm{rad}, t_{f}=10 \mathrm{~d} 19 \mathrm{~h} 13 \mathrm{~min}$.

Before going further, let us discuss the role played by $t_{f}$. We transformed the search for stable trajectories into a boundary value problem over a time span defined by $t_{f}$ that we apparently chose arbitrarily. By varying $t_{f}$, we notice that minima of the cost function correspond to different stable trajectories. In figure 13 we plot the cost function as a function of $\theta_{f}$ for $t=t_{f}-1 \mathrm{~h} 6 \mathrm{~min}=10 \mathrm{~d} 18 \mathrm{~h} 19 \mathrm{~min}$. In contrast to the previous case, the cost function has only one minimum at $\theta=3.814575 \mathrm{rad}$. In figure 14 we represent the trajectory that corresponds to this minimum. It is stable but different from the previous ones (figures 11 and 12). This result was expected and makes our approach even more valuable. Indeed, since we reduced the search for stable trajectories to a boundary value problem, we completely ignore the behavior of the spacecraft at intermediary times $t \in\left[0, t_{f}\right]$, we only take into account the states of the spacecraft at the initial time and at $t_{f}$. As a result, short term oscillations play a major role and alter the locus of the minima of $J$. Thus, by varying $t_{f}$ we are potentially able to find infinitely many stable trajectories going through $q_{0}$ at the initial time. This aspect allows us to design a deployment problem, for instance, where several spacecraft are at the same location at the initial time and we want to place them on stable trajectories that do not collide.

Furthermore, larger or smaller values of $t_{f}$ could have been chosen, however we must be aware that if $t_{f}$ is too small, short term oscillations may be as large as the drift and in that case the cost function does not discriminate well; its minima do not necessarily correspond to stable trajectories. On the other hand, if $t_{f}$ is very large, the minima correspond to $(M, T)$-stable relative trajectories with $T$ increasing as $t_{f}$ increases.

Finally, in the above example we selected trajectories that correspond to minima of $J$ and let $t_{f}$ vary to find several stable trajectories. However, trajectories that correspond to values of $J$ close to the minimum may be stable trajectories as well. If we vary $t_{f}$, say from $T^{1}$ to $T^{2}$, we noticed that the trajectory corresponding to the minimum of $J$ at $T^{1}$ is different from the one corresponding to the minimum of $J$ at $T^{2}$. Although the trajectory associated to $T^{1}$ does not correspond to a minimum of $J$ at $T^{2}$, it is stable and corresponds to 


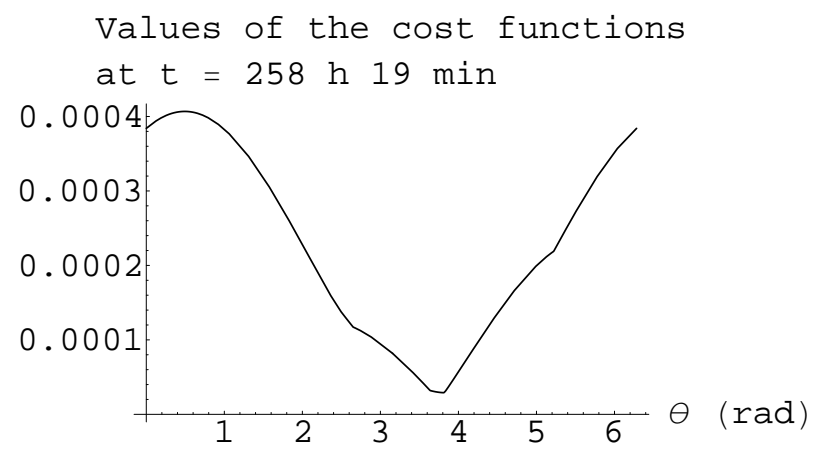

Figure 13. Cost function as a function of $\theta_{f}$

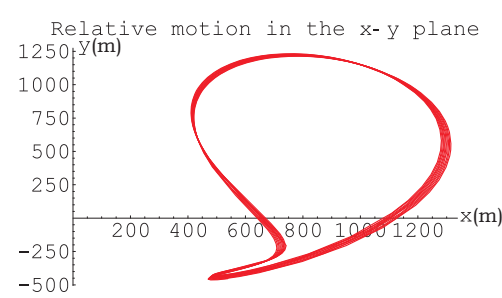

(a) $x-y$ motion during 26 hours

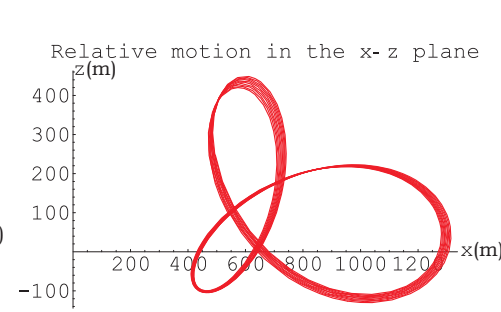

(b) $x-z$ motion during 26 hours
Relative motion in the $y^{-} z$ plane

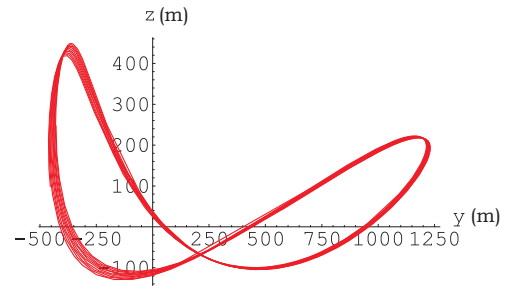

(c) $y-z$ motion during 26 hours

Figure 14. Trajectory associated with the minimum $\theta={ }_{f} 3.814575 \mathrm{rad}, t_{f}=10 \mathrm{~d} 18 \mathrm{~h} 19 \mathrm{~min}$.

a small value of $J$ at $T^{2}$. As a result, we are able to identify regions in which there are no stable trajectory that go through an initial position $q_{0}$ and through the circle of radius $r_{f}$ at $t_{f}$. For example, all stable trajectories that goes through $q_{0}=(495,-428.6,247.5) m$ and $q_{f}=700 \cos \left(\theta_{f}\right) \hat{f}_{1}+700 \sin \left(\theta_{f}\right) \hat{f}_{2} m$ at $t_{f}$ are localized on the arc defined by $\theta_{f} \in[0, \pi]$ when $t_{f}=10 d 19 \mathrm{~h} 13 \mathrm{~min}$ and by $\theta_{f} \in[2,5] \mathrm{rad}$ when $t_{f}=10 d 18$ h 19 min.

\section{Stable configurations}

In this section, we generalize the approach introduced above in order to design stable configurations. Without loss of generality, and for sake of simplicity, we assume that the formation is on $\mathcal{C}_{t_{0}}^{r_{0}}$ at the initial time so that the positions of the spacecraft are determined by the angle $\theta_{0}$, the angle between $\hat{f}_{1}$ and the local relative position vector. As a result, the initial position may be regarded as a function of $\theta_{0}$. Thus, Eqs. (21) and (22) provide a polynomial approximation of $p_{0}$ and $p_{f}$ in the variables $\left(\theta_{0}, \theta_{f}\right)$ (instead of $\theta_{f}$ only) with time-dependent coefficients. The procedure to find stable trajectories is the same as before but now we have an additional variable, $\theta_{0}$. In figure 15 we represent the values of the cost function as a function of $\theta_{i}$ and $\theta_{f}$ for different times. We notice that if two out of the three variables $\left(\theta_{f}, \theta_{0}, t_{f}\right)$ are given, there exists a value of the third variable that minimizes the cost function. In other words, whatever $\theta_{0}$ and $t_{f}$ are, there exists a stable trajectory that goes through the initial position at the initial time and reaches $\mathcal{C}_{t_{f}}^{r_{f}}$ in $t_{f}$ units of time. Moreover, if $t_{f}$ varies, minima of the cost function correspond to different stable trajectories due to short term oscillations.

\section{Example}

We consider a formation of 4 spacecraft equally spaced on a circle of radius $700 \mathrm{~m}$ about the reference trajectory that lies in the local vertical plane at the initial time. Spacecraft $k$ has its initial position defined by $\theta_{i}=\pi / 4+(k-1) \pi / 2$. Stable trajectories may be found by minimizing the cost function with respect to 


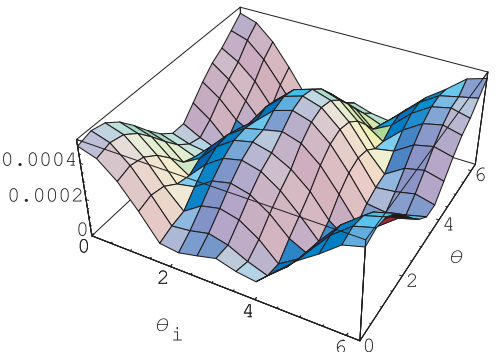

(a) At $t_{f}=10 d 18 \mathrm{~h} 19 \mathrm{~min}$

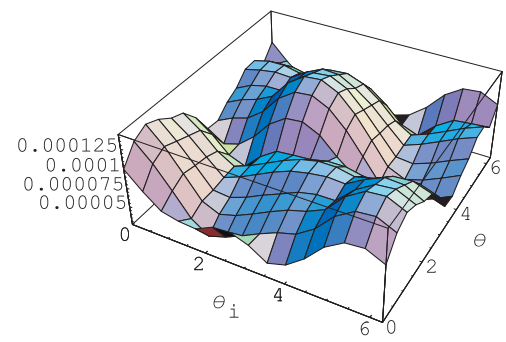

(d) At $t_{f}=10 d 18 \mathrm{~h} 50 \mathrm{~min}$

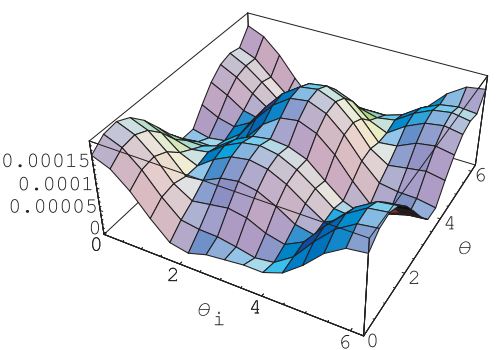

(b) At $t_{f}=10 d 18 \mathrm{~h} 34 \mathrm{~min}$

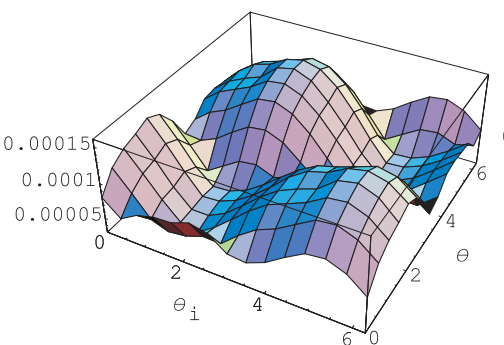

(e) At $t_{f}=10 d 18 h 57 \mathrm{~min}$

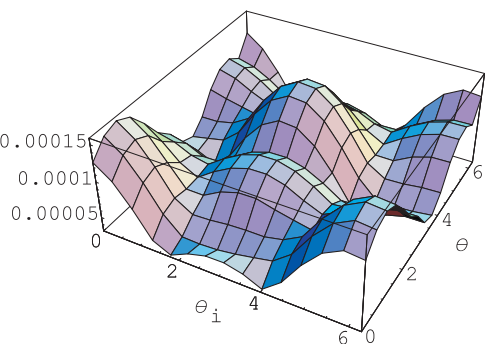

(c) At $t_{f}=10 d 18$ h $42 \mathrm{~min}$

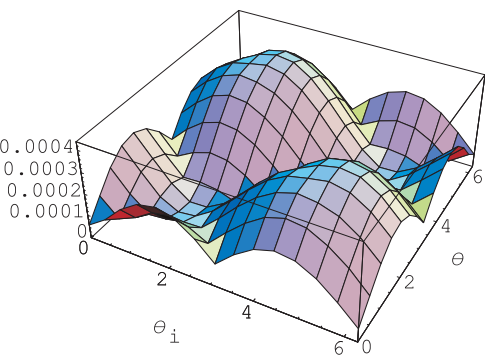

(f) At $t_{f}=10 d 19 h 13 \mathrm{~min}$

Figure 15. Cost function as a function of the initial and final positions for several $t_{f}$

$\theta$. For every choice of $t_{f}$ there is a solution to the minimization problem (see figure 15). As a result, we are able to find infinitely many stable trajectories for each spacecraft. In figure 16 we plot the trajectories of the four spacecraft that are found by considering $t_{f}=10 d 18 \mathrm{~h} 19 \mathrm{~m}$ and in figure $17, t_{f}=10 \mathrm{~d} 19 \mathrm{~h} 13 \mathrm{~m}$. The two solutions have very different properties; Even though the positions at the final time $t_{f}$ are constrained to be at $700 \mathrm{~m}$ from the reference trajectory in the local vertical plane, the relative distance may be large at intermediary times. For instance the solution found for $t_{f}=10 d 18 \mathrm{~h} 19 \mathrm{~m}$ yields a formation that is as large as $6 \mathrm{~km}$. Such trajectories cannot be found using linear approximations of the relative motion.

\section{Conclusions}

In this paper, we have joined elements of Hamilton-Jacobi theory and a robust algorithm that computes generating functions to address the challenges that arise in missions involving spacecraft flying in formation. Despite a complex dynamical model and an arbitrary reference trajectory we have been able to obtain a semi-analytic description of the phase flow as two-point boundary value problems. Such a description of the phase space is superior in many ways to the traditional approach based on the initial value problem. In particular it allows us to solve two-point boundary value problems using only simple functions evaluations. This aspect is crucial when dealing with spacecraft formations because of the combinatorial nature of the reconfiguration problem. In addition, we have shown that the algorithm we have developed is able to predict the dynamics over a long time span with high accuracy. The examples we have chosen illustrate the use of our method, but our method does not reduce to these examples and can be used to deal with more complex problems. To conclude, we recall the main feature of our method:

- The dynamical environment may be as complex as one wants, the only constraint being that the dynamical system must be Hamiltonian. In addition, the complexity of the dynamical system does not seriously impact the computation times.

- The reference trajectory may be arbitrary, however it influences the domain of convergence of the series defining $H^{h}$. Techniques to estimate this domain have been developed by Guibout and Scheeres. ${ }^{21}$

- The time span we consider may be very large, the larger it is the longer the ordinary differential equations obtained with the indirect algorithm should be integrated. The main advantage of describing the phase flow as two-point boundary value problems is that the time period we consider does not 
influence the accuracy of the results. This aspect is of main importance, especially as this is a weakness of traditional approaches based on the initial value problem.

- Our approach also allows one to deal with low-thrust spacecraft. In this case, the reconfiguration problem can be formulated as an optimal control problem whose necessary conditions for optimality are a Hamiltonian two-point boundary value problem. For these problems, the dynamical environment may not be Hamiltonian since the necessary conditions for optimality yield a Hamiltonian system. However, it should be emphasized that the dimensionality is double (because of the adjoint variables).

- There are no limitation on the complexity of the formation geometry in the reconfiguration problem as long as the geometry can be described with constraints on $(q, p)$ only.

- From the semi-analytic expression of the generating functions, several problems may be addressed. We have seen how to solve the reconfiguration problem and the deployment problem, we have also been able to find stable configurations and in previous articles the authors showed that one can also find periodic configurations. In the future, additional problems will be addressed.

Finally, the Mathematica $^{\circledR}$ package we have developed to run these simulations is freely available from the authors upon request.

\section{Acknowledgement}

The work described here was funded in part by NASA's Office of Space Science and by the Interplanetary Network Technology Program by a grant from the Jet Propulsion Laboratory, California Institute of Technology which is under contract with the National Aeronautics and Space Administration.

\section{A. Appendix: Computational times}

All the computations have been made using Mathematica ${ }^{\circledR} 5.0$ on a $2 G H z$ processor with $2 G B$ RAM running under Linux.

- Computation of the generating function $F_{1}$ up to order 4 over a time span of about 5 days: about 5 hours.

- Solving the 120 two-point boundary value problems in the first multi-task missions: about 3 minutes.

- Solving the second multi-task mission: about 1 minute.

- Solving the deployment problem: instantaneous once $F_{1}$ is known.

- Finding stable configurations: instantaneous once $F_{1}$ is known.

\section{References}

\footnotetext{
${ }^{1}$ Hope, A. S. and Trask, A. J., "Pulsed thrust method for hover formation flying," AAS/AIAA Astrodynamics Specialist Conference and Exhibit, Big Sky, Montana. Paper AAS 03-655, AAS, 2003.

${ }^{2}$ Vadali, S., Vaddi, S., and Alfriend, K., "An intelligent control concept for formation flying satellite constellations," International journal of robust and nonlinear control, Vol. 12, No. 2-3, 2002, pp. 97-115.

${ }^{3}$ Gurfil, P. and Kasdin, N. J., "Nonlinear Modeling and Control of Spacecraft Formation Dynamics in the Configuration Space," Submitted to the Journal of Guidance, Control and Dynamics, 2002.

${ }^{4}$ Scheeres, D., Hsiao, F., and Vinh, N., "Stabilizing motion relative to an unstable orbit: Applications to spacecraft formation flight," Journal of Guidance, Control, and Dynamics, Vol. 26, No. 1, 2003, pp. 62-73.

${ }^{5}$ Howell, K. and Marchand, B., "Control Strategies for Formation Flight in the Vicinity of a Libration Point Orbit," AIAA 13th AAS/AIAA Space Flight Mechanics Meeting, Ponce, Puerto Rico, AAS Paper No. 03-113, 2003.

${ }^{6}$ Vadali, S., Bae, H., and Alfriend, K. T., "Control of Libration Point Satellite Formations," 14th Space Flight Mechanics Meeting, Maui, Hawaii, AAS Paper No. 034-161, 2004.

${ }^{7}$ Vaddi, S. S., Alfriend, K. T., and Vadali, S. R., "Sub-optimal formation establishment and reconfiguration using impulsive thrust," AAS/AIAA Astrodynamics Specialist Conference and Exhibit, Big Sky, Montana. Paper AAS 03-590, AAS, 2003.

${ }^{8}$ Alfriend, K. and Schaub, H., "Dynamics and control of spacecraft formations: challenges and some solutions," $A A S / A I A A$ Astrodynamics Specialist Conference and Exhibit, College Station, Texas. Paper AAS 00-259, AAS, 2000.
} 
${ }^{9}$ Lovell, T., Horneman, K., Tollefson, M., and Tragesser, S., "A guidance algorithm for formation reconfiguration and maintenance based on perturbed Clohessy-Wiltshire equations," AIAA/AAS Astrodynamics Specialist Conference and Exhibit, Big Sky, Montana. Paper AAS 03-649, AAS, 2003.

${ }^{10}$ Hsiao, F. and Scheeres, D., "Design of Spacecraft Formation Orbits Relative to a Stabilized Trajectory," AAS/AIAA Space Flight Mechanics meeting, Ponce, Puerto Rico. Paper AAS 03-175, AAS, 2003.

${ }^{11}$ Hussein, I., Scheeres, D., and Hyland, D., "Control of a Satellite Formation For Imaging Applications," American Control Conference, Denver, Colorado, AAS, 2003.

${ }^{12}$ Mishne, D., "Maintaining periodic relative trajectories of satellite Formation by using power-limited thrusters," AAS/AIAA Astrodynamics Specialist Conference and Exhibit, Big Sky, Montana. Paper AAS 03-656, AAS, 2003.

${ }^{13}$ Alfriend, K. T., Vaddi, S. S., and Lovell, T. A., "Formation maintenance for low Earth near-circular orbits," $A A S / A I A A$ Astrodynamics Specialist Conference and Exhibit, Big Sky, Montana. Paper AAS 03-652, AAS, 2003.

${ }^{14}$ Koon, W., Marsden, J., Masdemont, J., and Murray, R., " $J_{2}$ dynamics and formation flight," AIAA Guidance, Navigation, and Control Conference, Montreal, Canada, August, AIAA 2001-4090, AIAA, 2001.

${ }^{15} \mathrm{Xu}, \mathrm{Y}$. and Fitz-Coy, N., "Genetic algorithm based sliding method control in the leader / follower satellites pair maintenance," AAS/AIAA Astrodynamics Specialist Conference and Exhibit, Big Sky, Montana. Paper AAS 03-648, AAS, 2003.

${ }^{16}$ Avanzini, G., Biamonti, D., and Minisci, E. A., "Minimum-fuel/minimum-time maneuvers of formation flying satellites," AAS/AIAA Astrodynamics Specialist Conference and Exhibit, Big Sky, Montana. Paper AAS 03-654, AAS, 2003.

${ }^{17}$ Wang, P. K. C. and Hadaegh, F. Y., "Minimum-fuel formation reconfiguration of multiple free-flying spacecraft," The Journal of the Astronautical Sciences, Vol. 47, No. 1-2, 1999, pp. 77-102.

${ }^{18}$ Guibout, V. M. and Scheeres, D. J., "Solving two-point boundary value problems using generating functions: Theory and Applications to optimal control and the study of Hamiltonian dynamical systems," Submitted to the Journal of nonlinear science, 2004.

${ }^{19}$ Chobotov, V. A., Orbital mechanics, American Institute of Aeronautics and Astronautics, 3rd ed., 2003.

${ }^{20}$ Guibout, V. M. and Scheeres, D. J., "Formation Flight with generating functions: Solving the relative boundary value problem," AIAA/AAS Astrodynamics Specialist Conference and Exhibit, Monterey, California. Paper AIAA 2002-4639, AIAA, 2002.

${ }^{21}$ Guibout, V. M. and Scheeres, D. J., "Solving relative two-point boundary value problems: Spacecraft formation flight transfers application," AIAA, Journal of Control, Guidance and Dynamics, Vol. 27, No. 4, 2003, pp. 693-704.

${ }^{22}$ Guibout, V. M. and Scheeres, D. J., "An algorithm that solves the Hamilton-Jacobi equation," In preparation, 2004.

${ }^{23}$ Guibout, V. M. and Scheeres, D. J., "Periodic orbits from generating functions," AAS/AIAA Astrodynamics Specialist Conference and Exhibit, Big Sky, Montana. Paper AAS 03-566, AAS, 2003.

${ }^{24}$ Scheeres, D. J., Park, C., and Guibout, V. M., "Solving optimal control problems with generating functions," AAS/AIAA Astrodynamics Specialist Conference and Exhibit, Big Sky, Montana. Paper AAS 03-575, AAS, 2003.

${ }^{25}$ Greenwood, D. T., Classical Dynamics, Prentice-Hall, 1977.

${ }^{26}$ Goldstein, H., Classical Mechanics, Addison-Wesley, 2nd ed., 1980.

${ }^{27}$ Arnold, V. I., Mathematical Methods of Classical Mechanics, Springer-Verlag, 2nd ed., 1988.

${ }^{28}$ Abraham, R. and Marsden, J. E., Foundations of mechanics, W. A. Benjamin, 2nd ed., 1978.

${ }^{29}$ Guibout, V. and Bloch, A., "Discrete variational principles and Hamilton-Jacobi theory for mechanical systems and optimal control problems," Physica D, submitted, 2004. 


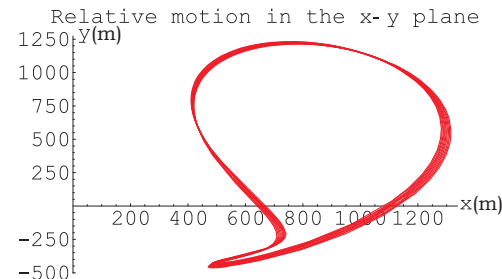

(a) Spacecraft 1

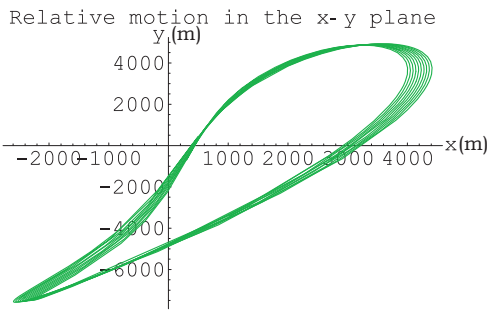

(d) Spacecraft 2

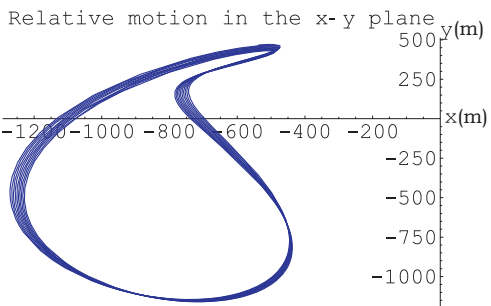

(g) Spacecraft 3

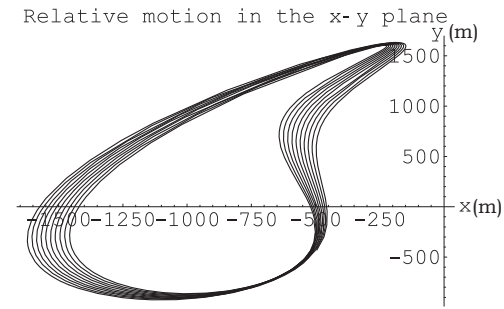

(j) Spacecraft 4

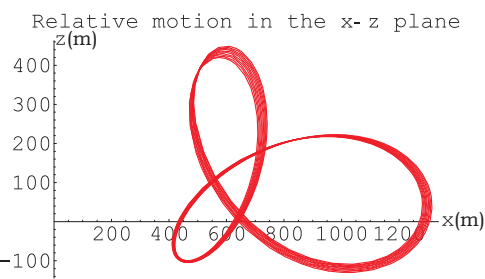

(b) Spacecraft 1

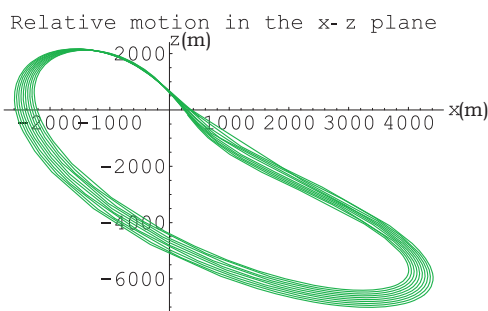

(e) Spacecraft 2

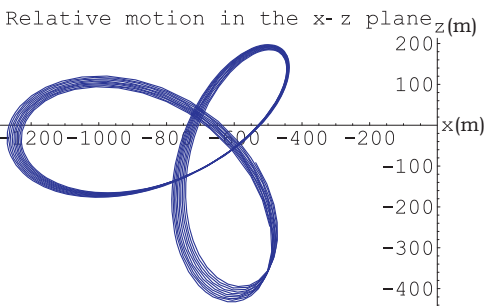

(h) Spacecraft 3

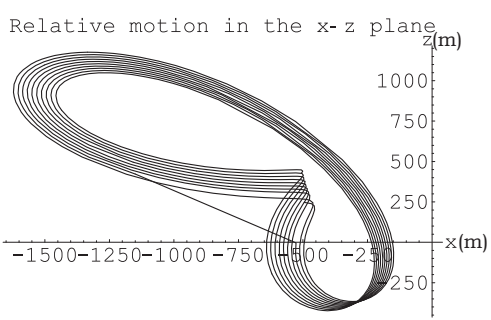

(k) Spacecraft 4

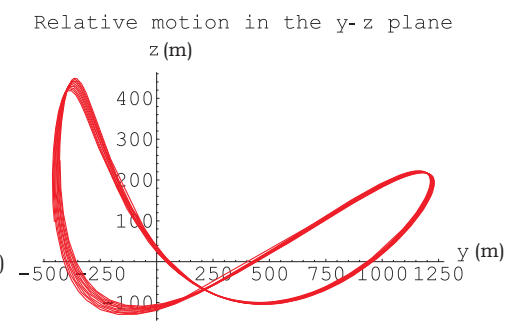

(c) Spacecraft 1

Relative motion in the $y^{-} \mathrm{z}$ plane

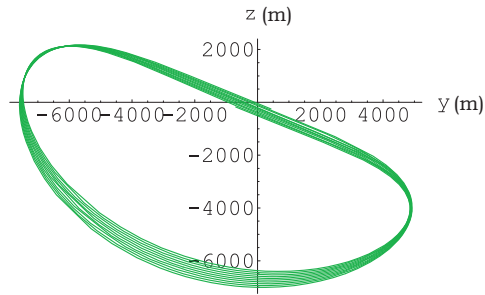

(f) Spacecraft 2

Relative motion in the $y-z$ plane

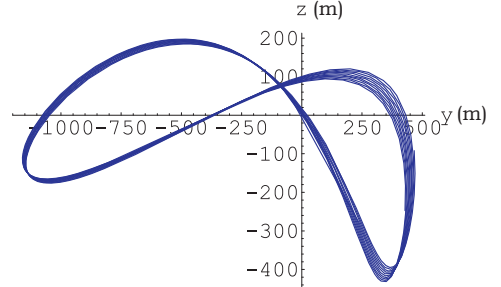

(i) Spacecraft 3

Relative motion in the $y^{-z}$ plane

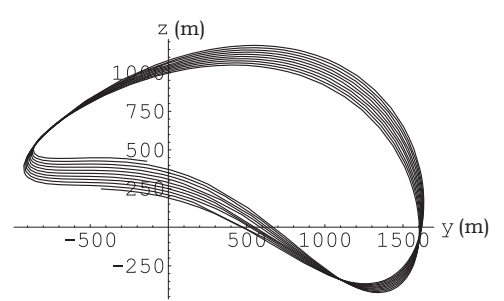

(1) Spacecraft 4

Figure 16. Trajectories of the four spacecraft found by minimizing the cost function at $t_{f}=10 \mathrm{~d} 18 \mathrm{~h} 19 \mathrm{~m}$ 


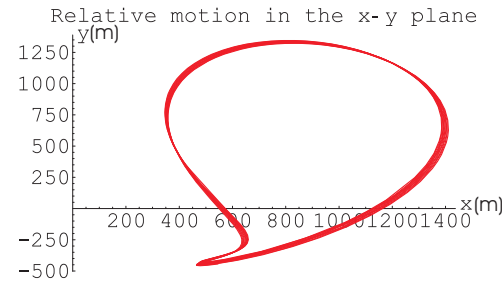

(a) Spacecraft 1

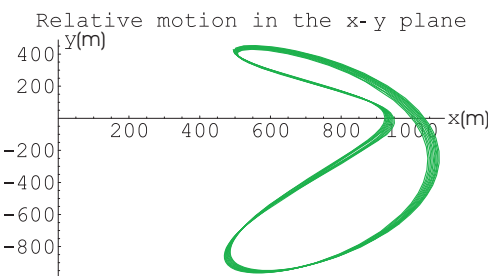

(d) Spacecraft 2

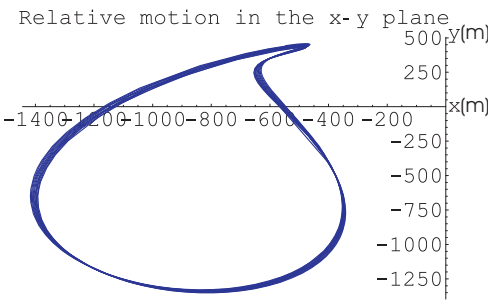

(g) Spacecraft 3

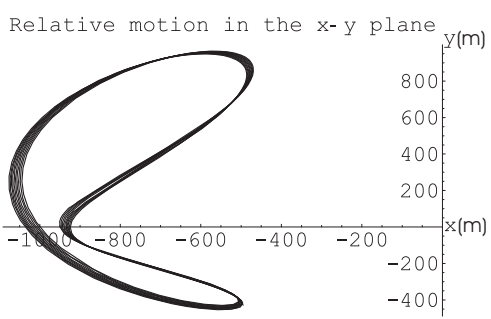

(j) Spacecraft 4

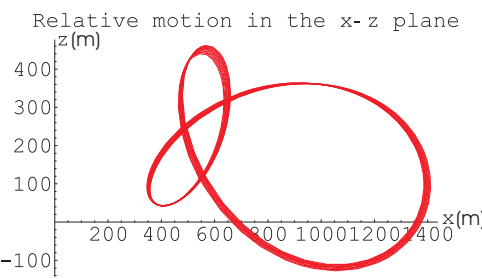

(b) Spacecraft 1

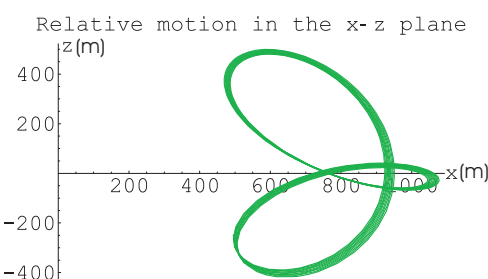

(e) Spacecraft 2

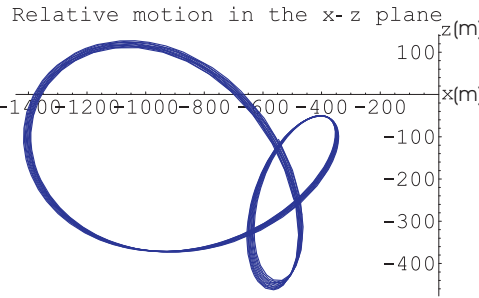

(h) Spacecraft 3

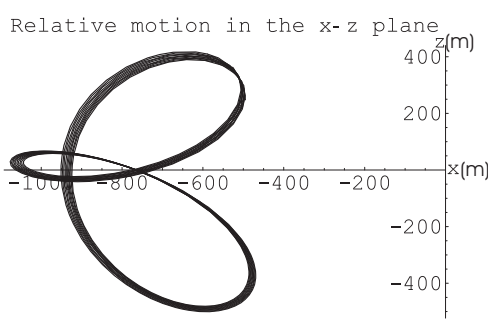

(k) Spacecraft 4

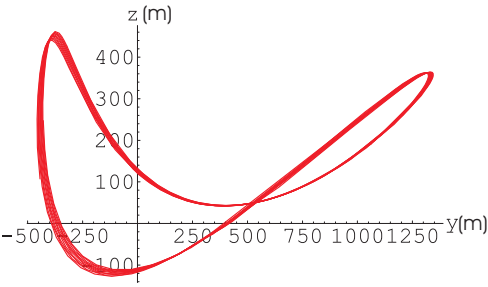

(c) Spacecraft 1

Relative motion in the $\mathrm{y}-\mathrm{z}$ plane

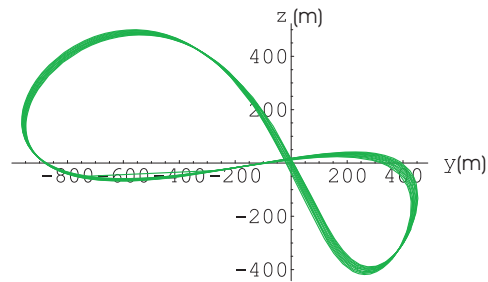

(f) Spacecraft 2

Relative motion in the $\mathrm{y}^{-} \mathrm{z}$ plane

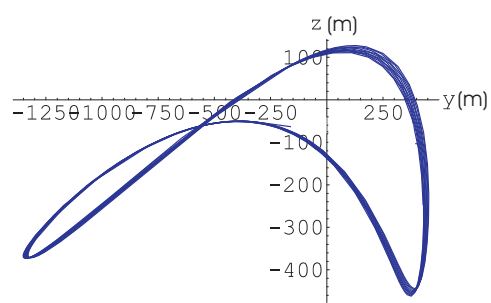

(i) Spacecraft 3

Relative motion in the $\mathrm{y}$ - $\mathrm{z}$ plane

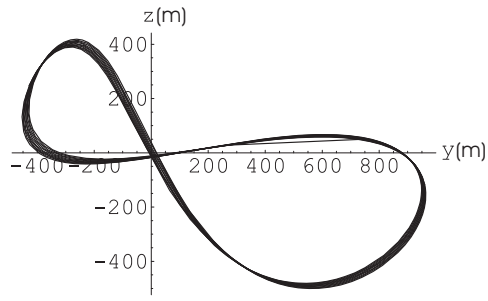

(l) Spacecraft 4

Figure 17. Trajectories of the four spacecraft found by minimizing the cost function at $t_{f}=10 \mathrm{~d} 19 \mathrm{~h} 13 \mathrm{~m}$ 